The Astrophysical JournaL, 540:342-361, 2000 September 1

(C) 2000. The American Astronomical Society. All rights reserved. Printed in U.S.A.

\title{
THE PROPAGATION OF MAGNETOCENTRIFUGALLY LAUNCHED JETS. I.
}

\author{
A. Frank, ${ }^{1}$ Thibaut Lery, ${ }^{2}$ T. A. Gardiner, ${ }^{3}$ T. W. Jones, ${ }^{4}$ AND D. RyU ${ }^{5}$ \\ Received 1999 September 10; accepted 2000 March 13
}

\begin{abstract}
We present simulations of the propagation of magnetized jets. This work differs from previous studies in that the cross-sectional distributions of the jets's state variables are derived from analytical models for magnetocentrifugal launching. The source is a magnetized rotator whose properties are specified as boundary conditions. The jets in these simulations are considerably more complex than the "top-hat" constant density, etc. profiles used in previous work. We find that density and magnetic field stratification (with radius) in the jet leads to new behavior including the separation of an inner jet core from a low density collar. We find this "jet within a jet" structure, along with the magnetic stresses, leads to propagation behaviors not observed in previous simulation studies. Our methodology allows us to compare MHD jets from different types of sources whose properties could ultimately be derived from the behavior of the propagating jets.
\end{abstract}

Subject headings: ISM: jets and outflows - magnetic fields - MHD

\section{INTRODUCTION}

Highly collimated supersonic jets are a ubiquitous phenomena occurring in many astrophysical environments. These jets are observed propagating from sources as diverse as active galactic nuclei (AGNs; Leahy 1991), young stellar objects (YSOs; Reipurth 1997), and Planetary Nebulae (PNe, Soker \& Livio 1994). While considerable progress has been made in understanding the nature of jets from AGNs and YSOs, there remains considerable debate concerning the nature of the more recently discovered $\mathrm{PNe}$ jets.

The ubiquity of jets in astrophysics has made them a popular subject for study. They are excellent laboratories for the study of basic astrophysical processes (shocks, instabilities, etc.). Their long dynamical or "look-back" times, $t_{\mathrm{dyn}}=L_{j} / V_{j}$, also make them ideal astrophysical fossils for studying the evolution of the obscured and often unobservable central sources, i.e., there is the hope in jet studies that the physics of the central engine can be revealed by studying the exhaust. Given the diversity of jet producing environments there also exists the hope that an underlying unity can be found in terms of the fundamental processes that create jets. Articulating these processes is one of the critical issues facing astrophysical jet studies.

Accretion disks are believed to play a key role in the physics of both YSOs and AGNs. In-falling, rotating matter is stored in these disks until dissipation allows material to spiral inward and feed the central, gravitating object. Both YSO and AGN disks are believed to support strong, wellordered magnetic fields. The current consensus holds that these fields are the agents for producing jets in a process known as "magnetocentrifugal launching." In this mechanism, plasma in the disk is loaded on to corotating field

\footnotetext{
${ }^{1}$ C. E. K. Mees Observatory, and (for editorial correspondence only) Department of Physics and Astronomy, University of Rochester, Rochester, NY 14627-0171; afrank@pas.rochester.edu.

${ }^{2}$ Department of Physics, Queen's University, Kingston, ON K7L 3N6, Canada.

${ }^{3}$ Department of Physics and Astronomy, University of Rochester, Rochester, NY 14627-0171; gardiner@pas.rochester.edu.

${ }^{4}$ Department of Astronomy, University of Minnesota, Minneapolis, MN 55455; twj@msi.umn.edu.

${ }^{5}$ Department of Astronomy and Space Science, Chungnam National University, Daejeon 305-764, Korea; ryu@sirus.chungnam.ac.kr.
}

lines. If conditions in the disk are favorable (i.e., field strength and orientation) the plasma is centrifugally flung outward along the field lines. Strong toroidal field components are generated in the flow as the field is dragged backward by the plasma inertia leading to collimation of the wind into a narrow jet. We note, however, that the external medium might also help focus the outflow. Magnetocentrifugal launching has been studied in detail by many authors both analytically (Heyvaerts \& Norman 1989; Pudritz 1991; Shu et al. 1994; 1995; Lery et al. 1999b) and through numerical simulations (Ouyed \& Pudritz 1997; Romanova et al. 1998; Kudoh et al. 1998).

In the YSO community two principle flavors of the magnetocentrifugal launching model exist. The first is a pure disk wind model (Pudritz 1991) in which the jet is generated at the surface of a Keplerian disk. The second, called " $\mathrm{X}$ winds" (Shu et al. 1994), produces a jet from the boundary layer between the disk and the central star's magnetosphere. Other models exist as well (Goodson et al. 1997) and there remains considerable debate as to which mechanisms are obtained in real YSO flows.

While there is an exhaustive literature concerning jet launching and collimation, there has also been considerable study of jet propagation. Propagation studies focus on scales many orders of magnitude larger (Reipurth 1997) than the region where collimation occurs. For example in the work of (Ouyed \& Pudritz 1997) the collimation of the jet was followed out to a height above the disk of $H=80 R_{i}$, where $R_{i}$ is the inner disk radius. Since $R_{i} \leq 10 R_{*}\left(R_{*}\right.$ is the stellar radius; Hartmann 1998), the scale of the simulation was at least 10 times smaller than the smallest scales on which jets have been resolved and at least $10^{3}$ times smaller than the typical scale of observational jet studies. Much of the propagation work has been numerical and for both YSOs and AGNs much of it has been have been purely hydrodynamic. For YSOs only a handful of MHD studies of jet propagation have been carried out to date (Todo et al. 1992; Cerqueira et al. 1998; Frank et al. 1998; Cerqueira et al. 1999; Gardiner et al. 2000; Stone \& Hardee 2000). If, however, strong magnetic forces produce the jets then these forces should effect their propagation downstream. Unless the fields are somehow removed, Maxwell stresses should alter at least some characteristics of the jet's propagation. 
Recently Frank et al. (1999) have shown that ambipolar diffusion may be operative in YSO jets in some part of the flow. However the timescales involved are such that changes in jet magnetic fields will only occur for parsecscale jets. Flows on timescales less than $\tau \approx 10^{3} \mathrm{yr}$ will not lose their fields. In the case of AGNs, the ambipolar timescales are even larger. Thus, a proper accounting for the MHD forces in the propagation of both YSO and AGN jets is needed. In this paper we focus mainly on YSOs but our results will be applicable to AGN jets as well.

To date all radiative MHD jet simulations of steady, constant density "top-hat" jets have been performed using simple field geometries. Cerqueira et al. (1999) showed that jets with purely poloidal $\boldsymbol{B}=B_{z} \boldsymbol{k}$ topologies did not have propagation characteristics that differed significantly from pure hydrodynamic jets. Gardiner et al. (2000) have also found similar results for pulsed "top-hat" MHD jets with poloidal fields. Frank et al. (1998), however, found that if the field had a strong toroidal $\left(B_{\phi}\right)$ component then the jet head could be strongly effected by the Maxwell stresses leading to the production of so-called nose cones. Nose cones form when postshock gas is restricted from lateral expansion by the axially directed "hoop stresses" associated with strong toroidal fields. Instead of back-flowing to form a cocoon, the shocked gas is confined to the head of the beam in the region downstream of the jet shock.

The hoop stresses lead to a conical streamlined configuration for the head i.e., a nose cone. Such structures were also seen in the early MHD simulations of AGN jets (Lind et al. 1989). In Frank et al. (1998) the addition of radiative losses, appropriate for YSO jets, caused the nose cones to narrow significantly. In a more extensive set of calculations, Stone \& Hardee (2000) found that MHD effects on jet propagation is strongly dependent on initial field topology.

While these results were promising, there still remains considerable distance to be traveled in the study of MHD jets. The principle issue that must be addressed is that all the simulations carried out to date is the use of ad hoc field topologies. Unless a force-free configuration is adopted, $\boldsymbol{J} \times \boldsymbol{B}=0$, Maxwell stresses will act on the jet beam independent of propagation effects. Thus some effort must be expended in developing equilibrium configurations for MHD jet simulation initial conditions. With little to guide them, all modelers have chosen simple topologies that allow for a simple specification of the required equilibrium. Frank et al. (1999) used a pure toroidal geometry. Gardiner et al. (2000) used a pure poloidal geometry. Cerqueira et al. (1999) used both toroidal and poloidal as well as force-free helical configurations, which had to extend throughout the entire computational domain (jet + ambient medium). Stone \& Hardee (2000) used helical pressure matched beams in a variety of configurations. None of the configurations used in these papers deviated from the simple constant velocity, constant density model for the jet beam. These efforts were necessary for articulating the basic role of MHD forces in jets, but they do not help establish a connection between conditions in the jet and the protostellar source (a protostar and rotating magnetized accretion disk). What is needed for use by the broader community is to begin the simulations with jet cross sections derived directly from magnetocentrifugal flow models. That is the goal of the work presented here.

In what follows we present models of MHD jet propagation with initial configurations in the jet taken directly from the solution of force balance perpendicular (the GradShafranov equation) and parallel (the Bernoulli equation) to magnetic surfaces generated by a magnetized rotator. Our simulations follow the evolution of jets composed of helical fields embedded in hypersonic plasmas whose density and velocity vary with radius. Thus our models constitute a further step toward realism in the theoretical description of magnetized astrophysical jets. The goal of this paper is to articulate the basic physics that can occur in these kinds of jets and to look for differences between the propagation of jets forming from different kinds of rotators. We note that the parameter space of solutions is quite large and in this paper we present only the first results of this project. In future papers we will present a more systematic exploration of parameter space.

The plan of the paper is as follows. In $\S 2$ we describe the methods used to construct the initial equilibria and numerically simulate the flows. In $\S 3$ we present results of our simulations focusing on adiabatic, isothermal and radiative cases. The next section compares the results with observations. Finally, in $\S 5$ we present and discuss our conclusions.

\section{NUMERICAL METHODS AND INITIAL EQUILIBRIA}

\subsection{Basic Equations}

We numerically integrate the equations of ideal magnetohydrodynamics (MHD), modified to include the loss of thermal energy due to optically thin radiative losses. In cylindrical coordinates these equations take the following form,

$$
\begin{gathered}
\frac{\partial \rho}{\partial t}+\frac{1}{r} \frac{\partial}{\partial r}\left(r \rho v_{r}\right)+\frac{\partial}{\partial z}\left(\rho v_{z}\right)=0 \\
\frac{\partial \rho v_{r}}{\partial t}+\frac{1}{r} \frac{\partial}{\partial r}\left(r \rho v_{r}^{2}-r B_{r}^{2}\right) \\
+\frac{\partial}{\partial r}\left(p^{*}\right)+\frac{\partial}{\partial z}\left(\rho v_{r} v_{z}-B_{r} B_{z}\right)=\frac{\left(\rho v_{\phi}^{2}-B_{\phi}^{2}\right)}{r} \\
\frac{\partial \rho v_{\phi}}{\partial t}+\frac{1}{r} \frac{\partial}{\partial r}\left(r \rho v_{\phi} v_{r}-r B_{\phi} B_{r}\right) \\
+\frac{\partial}{\partial z}\left(\rho v_{\phi} v_{z}-B_{\phi} B_{z}\right)=\frac{\left(B_{r} B_{\phi}-\rho v_{\phi} v_{r}\right)}{r} \\
\frac{\partial \rho v_{z}}{\partial t}+\frac{1}{r} \frac{\partial}{\partial r}\left(r \rho v_{z} v_{r}-r B_{z} B_{r}\right)+\frac{\partial}{\partial z}\left(\rho v_{z}^{2}-B_{z}^{2}+p^{*}\right)=0 \\
\frac{\partial B_{r}}{\partial t}+\frac{\partial}{\partial z}\left(v_{z} B_{r}-v_{r} B_{z}\right)=0 \\
+\frac{1}{r} \frac{\partial}{\partial r}\left(r v_{r} B_{\phi}-r v_{\phi} B_{r}\right) \\
+\frac{\partial}{\partial z}\left(v_{z} B_{\phi}-v_{\phi} B_{z}\right)=\frac{\left(v_{r} B_{\phi}-v_{\phi} B_{r}\right)}{r} \\
\frac{\partial B_{z}}{\partial t}+\frac{1}{r} \frac{\partial}{\partial r}\left(r v_{r} B_{z}-r v_{z} B_{r}\right)=0
\end{gathered}
$$




$$
\begin{aligned}
\frac{\partial E}{\partial t}+ & \frac{1}{r} \frac{\partial}{\partial r}\left[r\left(E+p^{*}\right) v_{r}-r B_{r}(\boldsymbol{B} \cdot \boldsymbol{v})\right] \\
& +\frac{\partial}{\partial z}\left[\left(E+p^{*}\right) v_{z}-B_{z}(\boldsymbol{B} \cdot \boldsymbol{v})\right]=-\left(\frac{\rho}{\mu}\right)^{2} \Lambda(T),
\end{aligned}
$$

The total energy and pressure are given by

$$
\begin{aligned}
p^{*} & =p+\frac{1}{2} B^{2}, \\
E & =\frac{1}{2} \rho v^{2}+\frac{p}{\gamma-1}+\frac{1}{2} B^{2},
\end{aligned}
$$

where $\mu$ is the mean molecular weight and $B^{2}=\boldsymbol{B} \cdot \boldsymbol{B}$. Equations (1) and (8) represents conservation of mass and energy, respectively. Equations (2)-(4) represent conservation of momentum. Equations (5)-(7) represent the induction equation. The energy conservation equation includes a source term, $n^{2} \Lambda(T)$, (where $n=\rho / \mu$ is the number density), which models radiative losses in the optically thin limit. We use the Dalgarno-McCray "coronal" cooling curve (Dalgarno et al. 1972). A "floor" temperature of $T_{f}=10^{4} \mathrm{~K}$ is set such that gas can not cool to lower values. The fluid is assumed to be an ideal gas, where $\gamma$ is the ratio of specific heats. Other relevant quantities are the sound speed $c=(\gamma p / \rho)^{1 / 2}$, the Alfvén speed parallel to the magnetic field $v_{a}=\left(B^{2} / \rho\right)^{1 / 2}$ and the plasma beta parameter, $\beta=2 p / B^{2}$.

In addition to the hyperbolic equations represented above an additional constraint is imposed via the condition of flux conservation,

$$
\frac{1}{r} \frac{\partial}{\partial r} B_{r}+\frac{\partial}{\partial z} B_{z}=0 .
$$

Using these equations we model the propagation of a magnetized jet through a constant density, constant pressure magnetized ambient medium. The initial conditions for the jet, i.e., its cross-sectional distribution of $\rho, p, v$, and $\boldsymbol{B}$, are calculated via the Given Geometry Method of (Lery et al. 1998; 1999b; Lery \& Frank 2000). We describe this method and the equilibrium MHD jet solutions it produces in the next section.

\subsubsection{The Model}

The jets we inject into the computational grid are taken directly from a (simplified) model of the magnetocentrifugal launching/collimation process. The model, known as the Given Geometry Method (GGM: Lery et al. 1998, 1999b, Lery \& Frank 2000) allows asymptotic MHD jet equilibria to be linked directly to the properties of a rotating source. The GGM assumes a time-independent, axisymmetric flow. It further simplifies the problem of magnetocentrifugal launching/collimation by assuming that the nested magnetic flux surfaces defining the flow (labeled by the variable a) possess a shape that is known a priori inside the fast critical surface. The fast surface defines the locus of points beyond which the flow is kinetic energy-dominated. The flux surfaces are assumed to be conical and, as an additional simplification, an equilibrium across the surfaces is assumed at the Alfvén point, which yields an equation referred to as the Alfvén regularity condition. This condition is not a criticality condition since the Alfvén point is not strictly a critical point.

The flow properties must be determined by solving for the equilibrium of forces parallel and perpendicular to the magnetic surfaces (the former described by using the
Bernoulli equation for a polytropic equation of state and the latter is solved via the Grad-Shafronov equation). The equilibrium parallel to the surfaces takes the form of criticality conditions at the two other (fast and slow) MHD critical points. This corresponds to differential form of the Bernoulli equation on constant $a$ with respect to $\rho$ and $r$ vanishing at the critical points.

In the general case the GGM yields five integrals of motion that are preserved on any axisymmetric magnetic surface $a$. Two of the integrals are given as boundary conditions in the model. These are the angular velocity $\Omega(a)$ and an entropy (or ploytropic) factor $Q(a)$. These are supplied as a model for the source rotator. We note that the entropy parameter $Q(a)$ can be described as follows: The density $\rho$ is related to the pressure $p$ by a polytropic equation of state, $p=Q(a) \rho^{\gamma}$, where $\gamma$ is the polytropic index and $Q$ the polytropic constant that is related to the entropy. This assumption replaces consideration of energy balance and is meant to simply represent more complex heating and cooling processes (see, e.g., Vlahakis \& Tsinganos 1998 for more general equations of state). Changing $Q(a)$ changes the local thermal energy balance in the flow.

The Alfvén regularity condition together with the criticality conditions then determine the three other unknown integrals: namely the specific energy $E(a)$; the specific angular momentum $L(a)$; the mass to magnetic flux ratio $\alpha(a)$. Far from the source (large $z$ ) the flow becomes cylindrically collimated. In this asymptotic regime the jet is assumed to be in pressure equilibrium with an external medium. The pressure matching condition along with the Grad-Shafronov and Bernoulli equations are all solved in the asymptotic cylindrically collimated regime.

We note that in the GGM the source (e.g. the accretion disk) is not explicitly described since it is pointlike. Instead the shape of the magnetic field lines defined by the flux function $a(r, z)$ is specified out to the fast magnetosonic point, but not its angular distribution. The rotation rate $\Omega(a)$ and the polytropic parameter $Q(a)$ are then specified on the field lines. The shape of the magnetic field lines from the fast magnetosonic point to the fully collimated region is not specified. The strong toroidal component that develops in the wind and the fully collimated jet develops mainly as a result of differential rotation and the inertia of mass on the field lines. This is similar to other disk wind models (Ouyed \& Pudritz 1997).

\subsubsection{Numerical Solutions}

Inside the fast critical surface, the variables calculated in the numerical procedure are the energy $E$ and the radii and densities at the three critical surfaces, $\left(r_{s}, r_{f}, r_{A}, \rho_{s}, \rho_{f}, \rho_{A}\right)$. In the asymptotic cylindrically collimated regime, the jet is entirely defined by this set of $r$ and $\rho$ and all other physical quantities can be derived from them. For the numerical calculations, the equations have been reformulated as ordinary differential equations or converted from algebraic conditions into ordinary differential equations as functions of the flux surfaces $a$. The system consists of eight differential equations and the numerical solutions are obtained by initiating the integration of the system from the axis. Given the input parameters $Q(a), \Omega(a), \alpha_{0}, \gamma$, and $\rho_{0}$, all the critical positions and densities can be numerically obtained using analytical formulae (see Lery et al. 1998). We further constrain the solution to be super-Alfvénic and superfastmagnetosonic on the axis in the asymptotic region. 


\subsubsection{Classes of Jet Equilibria}

In our approach the most important aspect of the source rotator is angular rotation profile. We focus on this aspect of the source because it most clearly connects to different scenarios of magnetocentrifugal launching/collimation. Profiles of angular velocity of the source rotator considered in this paper are shown in Figure 1. The pure Keplerian rotator (dashed line) starts with a constant rotation close to

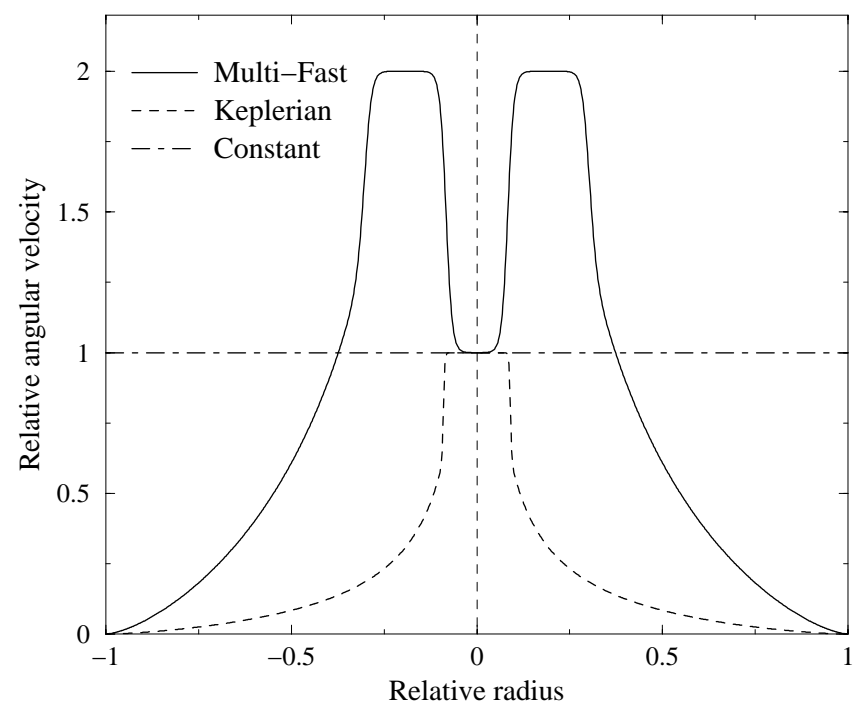

FIG. 1.-Rotation laws for pure Keplerian (dashed line) and multicomponent (solid line) models. Axial angular velocity is set to unity. the axis, as in the rigid body case (dot-dashed lines), but then follows a Keplerian profile. The multicomponent (solid line) case also starts with a rigid rotation corresponding, for example, to an axial ordinary wind. The angular velocity then doubles its value in order to model a jet rotating more rapidly than the star in an intermediate region between the ordinary wind and the Keplerian disc wind that follows. Note that the angular velocity is always sub-Keplerian in the intermediate region. For all the rotation laws, the axial value of the angular velocity $\Omega_{0}$ is set to unity in the figure, and the radius is normalized to the size of the jet. For reference we also show the profile of a solid body rotator though we will not consider this model in the simulations. Figure 2 also shows that a return poloidal electric current flows back inside the jet for both the Keplerian and the multicomponent jets.

As shown in Lery \& Frank (2000), it is possible to derive an approximate analytical solutions of the model in the cylindrical region. It has been found that the density can be expressed as a function of $r$ and of the first integrals as $\rho(r) \approx C \alpha(r) / \Omega(r) r^{2}$, where $C$ is a constant. The asymptotic poloidal velocity of the flow can also be derived and is given by $v_{z}(r) \approx \Omega(r) r / C$. Therefore the velocity increases with the angular velocity while the density decreases. This explains why the density drops when $\Omega$ is important in the inner part of the jet for the multicomponent case, while it increases afterward in the Keplerian rotation regime. The velocity roughly follows an opposite behavior with respect to the angular velocity. More detailed analysis of these equilibria are given by Lery $\&$ Frank (2000).
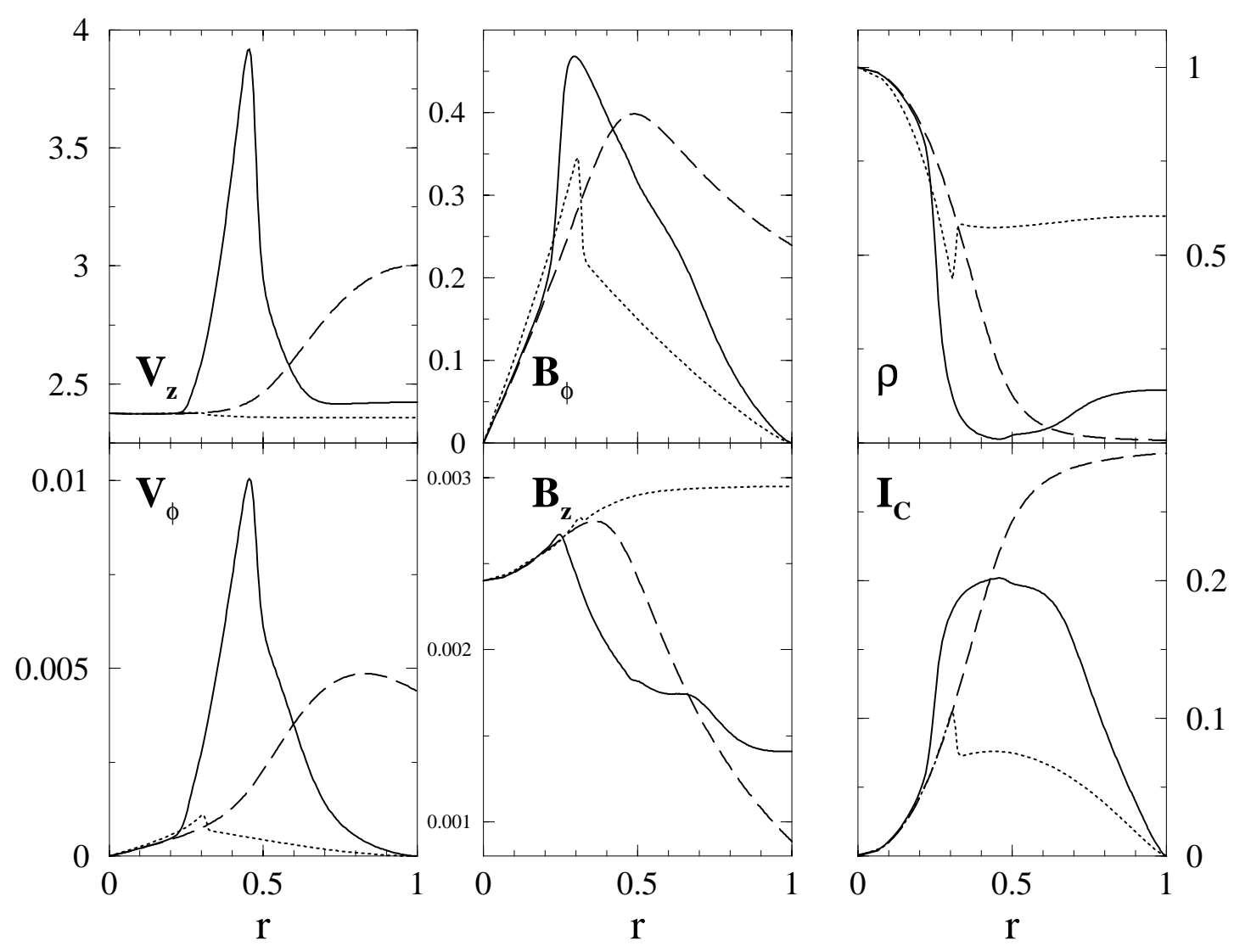

FIG. 2.-Variations with the relative radius of the velocity $V$ and magnetic field $B$ components, of the density $\rho$, and of the net electric current $I_{C}$ in the cylindrically collimated regime. Pure Keplerian (dashed line) and multicomponent (solid line) rotation laws are considered. 


\subsubsection{Identification of Basic Features}

The quantities that define the jet in the cylindrically collimated regime, are plotted in Figure 2 for pure Keplerian, multicomponent and constant rotations. The Keplerian and multicomponent models will be used as input for the numerical simulations. The $z$ and $\phi$ components of velocity and magnetic field are represented together with the density $\rho$ and the net electric current $I_{C}$, as functions of the relative radius (normalized to the jet radius). The length scale is the jet radius, the density is normalized to its value on the jet axis $\rho_{0}$, and the nondimensional velocities refer to the fast magnetosonic velocity $v_{f}^{2}=c_{s}^{2}+v_{A}^{2}$ on the axis, $c_{s}$ being the sound speed. The magnetic field is normalized to $\sqrt{\rho_{0}} v_{f}$.

The most important features in these graphs are the variations of the toroidal component of the magnetic field and of the density. Note that the region near $R=.3 R_{j}$ is dominated by the magnetic pinching force, or "hoop stress," where $B_{\phi}$ is maximum. The gas pressure is important at radii less than this value in order to maintain the equilibrium and is the origin of the large density gradients in this region. We denote the high-density region centered on the axis as the core, and the lower density outer regions as the collar. Note that the bulk of the jet's momentum resides in the core. Hence we expect this portion of the beam to penetrate more easily into the ambient medium during the jet's propagation, while the collar will be more strongly decelerated. Figure 2 also shows that a return poloidal electric current flows back inside the jet for both the Keplerian and the multicomponent jets. More detailed analysis of these equilibria are given by Lery \& Frank (2000).

Thus Keplerian and multicomponent jets are characterized by a dense, current-carrying core, carrying most of the momentum, and are surrounded by a collar carrying an internal return current.

\subsubsection{Scaling for the Simulations}

The input parameters of the model can be selected so as to qualitatively reproduce observed situations. Given the properties of the jet-emitting object, i.e., its radius $R_{*}$, its temperature $T_{*}$, the total mass-loss rate $\dot{M}_{*}$, the base density $n_{*}$, the magnetic field $B_{*}$, the factor $Q_{*}$ and $\gamma$, it is possible to deduce the dimensionless parameters $\bar{\Omega}, \bar{Q}, \bar{\alpha}_{0}$. The parameter $\bar{\alpha}_{0}$ can be a posteriori related to the massloss rate $\dot{M}_{*}, R_{*}$, and the magnetic field $B_{*}$. So we define $Q_{*} \equiv 2 k T_{*} n_{*} /\left(m_{p} n_{*}\right)^{\gamma}, \quad \alpha_{*} \equiv \dot{M}_{*} / 4 \pi R_{*}^{2} B_{*}, \quad$ and $\Omega_{*} \equiv$ $\left(G M_{*} / R_{*}^{3}\right)^{1 / 2}$. All those quantities are nondimensionalized to reference values by setting $\bar{Q} \equiv Q_{*} / Q_{\text {ref }}, \bar{\alpha}_{0} \equiv \alpha_{*} / \alpha_{\text {ref }}$ and $\bar{\Omega} \equiv \Omega_{*} / \Omega_{\text {ref }}$. The entropy $\bar{Q}(a)$ is assumed to be constant across the jet. In the present paper, we have chosen to model YSO jets with different rotation laws using typical values for T Tauri stars as presented by Bertout et al. (1988). At the base of flow, we deduce the corresponding dimensionless input parameters: $\bar{Q}=0.87, \bar{\Omega}=2, \bar{\alpha}_{0}=0.7$, and $\rho_{0}=5 \cdot 10^{-7}$. Major quantities of reference are then given (in cgs) by $R_{\text {ref }}=10^{15} \mathrm{~cm}, n_{\text {ref }}=250 \mathrm{~cm}^{-3}, v_{\text {ref }}=10^{7} \mathrm{~cm} \mathrm{~s}^{-1}$ for YSOs. $V_{r}$ ef is simply a canonical speed for YSO jets that we use to set the scales in the simulations.

\subsubsection{Comparisons with Other Models}

A detailed comparison of the present model with previously published studies has been given by Lery \& Frank (2000). Here we report only the most important conclusions.
As with Ferreira (1997), it is possible to show that the GGM yields a minimum mass-loss rate injected in the jet that has a lower limit and cannot be arbitrarily small. These results also agree with Ostriker (1997) and Lery et al. (1999a), who conclude that the optical jet may represent only the densest part of the total outflow. We obtain a fast magnetosonic Mach number (which also corresponds to the Alfvénic Mach number on the axis) between 2 and 4. This range corresponds to what (Camenzind 1997) has found for his model for low-mass protostellar object. The corresponding jets have low fast magnetosonic Mach numbers $M_{A} \simeq 2$. By taking into account an accretion disc around the stellar magnetosphere, Fendt \& Camenzind (1996) also find a fast magnetosonic Mach number to be 2.5. This results does not, however, appear to be a general statement about MHD jets since there exist models with larger values (Sauty \& Tsinganos 1994; Trussoni, Tsinganos, \& Sauty 1997). Finally, the analytical results given by Shu et al. (1995) agree with those of the GGM model in terms of jet structure. We note however that despite the similarity of the analytical results, neither the multicomponent (nor the Keplerian) case can be seen as equivalent to the $\mathrm{X}$-wind model. Note in particular that our model does not describe the physical processes occurring at the source itself, i.e., at the surface of the disk or the disk-star boundary.

\subsection{Numerical Method and Implementation}

A detailed description of the numerical code can be found in references given below. Here we simply state the code's most salient features. Specifically, the method we use to solve equations (1)-(8) is explicit, finite element (volume), upwinded, conservative, second-order accurate, and total variation diminishing (TVD). This refers to the ability of the code to capture strong discontinuities in the flow without producing spurious oscillations. TVD methods are part of a general class of "high-resolution" codes that solve the hydro or MHD equations in conservative form by using a Gudinov method (i.e., solving the Riemann problem at every grid interface) and including sophisticated algorithms for limiting the fluxes through cell boundaries to keep the solution monotone. More detail concerning high-resolution methods can be found in Leveque (1998). The code is conservative up to machine accuracy, ensuring that it will accurately capture shock strengths and speeds. It has been well tested in standard one-dimensional shock tube tests as well as multidimensional stability calculations. Various manifestations of the code have been reported in the literature including its one-dimensional Cartesian form (Ryu \& Jones 1995), its two-dimensional Cartesian form (Ryu, Jones, \& Frank 1995a), and its two-dimensionalaxisymmetric (cylindrical coordinates) form (Ryu et al. 1995b). The TVD property is ensured in the same way as was done originally by Harten for the Euler equations in (Harten 1983). In the two-dimensional versions of the code, multidimensionality is handled through the use of Strang splitting (Strang 1968). The cooling is applied in a first-order fashion. Finally, the crucial and problematic issue of maintaining $\boldsymbol{\nabla} \cdot \boldsymbol{B}=0$ is accomplished with a staggered grid approach (Ryu et al. 1998).

Each simulation was carried out in cylindrical coordinates $(r, \phi, z)$ with axisymmetry and inversion symmetry across the $z=0$ plane. We follow a quarter meridional plane $(r \geq 0, z \geq 0, \phi=0)$ with $512 \times 2048$ grid cells. The jet radius spans 64 grid cells. Thus our simulations follow 
the jet propagation for $Z=32 R_{j}$. In Table 1 we present a set of important physical parameters for the simulations presented below. While we will use dimensionless variables in most of the description that follows, Table 1 allows the reader to compare the physical scales in the simulations with observations.

In what follows we express all distances in terms of $R_{j}$ and all times in terms of the magnetosonic crossing time $\tau=R_{j} / M_{f}$, where $M_{f}=\left(v_{a}^{2}+c^{2}\right)^{1 / 2}$ (note: for our initial conditions the magnetosonic speed and the fast mode speed are identical). We utilize outflow boundary conditions at the outermost radial and axial boundaries. In cylindrical coordinates the $r=0$ line is necessarily a reflecting boundary. During the tests that we have run we have found little evidence of incorrect reflections from the $r=0$ line due to the coordinate singularity, though it must be admitted that this problem plagues all numerical codes in cylindrical coordinates with axisymmetry. Inversion symmetry through the $z=0$ plane dictates the use of reflecting boundary conditions at the $z=0$ plane. In some simulations we have found that waves propagating inward from the outermost radial boundary (caused by the bow shock propagating off the grid) led to a slow compression of field at the base of the jet at late times. We wished to avoid the use of logarithmic grids hence we suppressed the inflow of material at the outer radial boundaries problem by injecting a slow $\left(M_{s} \sim 1.2\right)$ wide-angle flow at the base of the grid. This advected the material off the grid and kept the field from being overly compressed near the jet inlet at the base of the grid and the imposed flow had no effect of the propagation of the jet far downstream.

In each simulation we inject the jet into the computational domain via 2 layers of "ghost-zones" below the base $z=0$ of the grid. The jet properties are read into the grid from data files provided by the GGM described above. The density and pressure in the ambient medium are copied from values in the last radial zone of the jet: $\rho_{a}=$ $\rho_{j}\left(R_{j}\right), p_{a}=p_{j}\left(R_{j}\right)$. In addition the ambient medium is given a pure poloidal magnetic field $\left(\vec{B}_{a}=B_{a, z} \hat{k}\right)$ whose magnitude is also taken from the last radial zone of the jet $B_{a, z}=$ $B_{j, z}\left(R_{j}\right)$. Since $B_{j, \phi}\left(R_{j}\right)=0$ our jets are in radial pressure balance the with the ambient medium.

In order to articulate the basic dynamics inherent to the flows, we have run three classes of model for both the Keplerian and multicomponent jet. In our adiabatic models we have set $\gamma=5 / 3$ and turned off the cooling source term. In our isothermal models we have set $\gamma=1.001$ and turned off the cooling term. In our radiative models $\gamma=5 / 3$ and the cooling source term was turned on. We have run both radiative and isothermal models as consistency checks as well as to allow us to model jets with different Mach numbers. For reasons explained above the equilibria pro- vided by the GGM yielded jets of low magnetosonic number $\left(3<M_{f}<5\right)$. We wish to model jets with lower temperatures. This could be accomplished by scaling down both the pressure and magnetic field such that the force balance was maintained. In this way we were able to model jets with Mach numbers of order $\left(6<M_{f}<9\right)$.

The cross-sectional variation of $\boldsymbol{B}_{j}(r)$ in the jet presents a problem in terms of initial conditions. This is a general difficulty that all attempts to model the evolution of magnetized jets must confront. Flux conservation, $\boldsymbol{\nabla} \cdot \boldsymbol{B}=0$, demands that any discontinuities in the field must be associated with current sheets (which are the cause of field kinks at MHD shocks). Attempts to initialize simulations of magnetized jets propagating into magnetized ambient media must deal with the likely mismatch of field topologies and magnitudes at the head and sides of the jet when the simulation is first switched on. The use of cylindrical coordinates eliminates the problem for the $B_{\phi}$ component. While an initial discontinuity in the toroidal component may produce transients, it will not violate flux conservation. Thus we must only deal with the $r$ - and $z$-components of the field. In our simulations we solved the problem by continuing the $z$-component of the jet field into the ambient medium. Thus for $r<R_{j}, B_{a, z}(r)=B_{j, z}(r)$. Since $B_{z}$ is relatively weak, $B_{z}^{2} / 2 \ll p$, the gradient in the ambient field produces little mass motion.

In order to test the effect of the initial conditions on the observed behavior (i.e., transients), we have run a series of models which began with the jet and ambient conditions joined smoothly via a hyperbolic tangent function. The smoothing length $h$ was varied from $h=.5 R_{j}$ to $h=9 R_{j}$. We found that the long term behavior of the jet was unaffected by the choice of $h$. We also note that this version of the code produces a relatively strong boundary layer at the jet/ambient gas interface at distances far from (well behind) the head of the jet. While such layers are to be expected owing to unresolved instabilities (mainly Kelvin-Helmholtz modes), we found the effect was partially attributable to the treatment of transverse wave modes in the code. We performed a number of tests to ensure that changes in the flow variables in the boundary layer were not affecting the results.

\section{RESULTS}

In this section we present the results of the simulations. We provide a description of the behavior seen in the models along with attempts to understand the underlying physics.

\subsection{Basic Features}

Several features are common to almost all of the simulations. In the input equilibria the core-collar structure is always present with gradients of jet variables between the

TABLE 1

SimUlation PARAMETERS

\begin{tabular}{cccccc}
\hline \hline Simulation & $\begin{array}{c}R_{j} \\
(\mathrm{~cm})\end{array}$ & $\begin{array}{c}n \\
\left(\mathrm{~cm}^{-3}\right)\end{array}$ & $\begin{array}{c}V_{j} \\
\left(\mathrm{~km} \mathrm{~s}^{-1}\right)\end{array}$ & $\begin{array}{c}\mathrm{T}_{j} \\
(\mathrm{~K})\end{array}$ & $\begin{array}{c}B_{\phi} \\
(\mathrm{G})\end{array}$ \\
\hline Adiabatic Keplerian ............... & $1.36 \times 10^{15}$ & 120 & 120 & $3.8 \times 10^{4}$ & $9.1 \times 10^{-5}$ \\
Radiative Keplerian ........... & $1.36 \times 10^{15}$ & 120 & 120 & $1.0 \times 10^{4}$ & $4.5 \times 10^{-5}$ \\
Adiabatic multicomponent ....... & $1.68 \times 10^{16}$ & 120 & 190 & $3.8 \times 10^{4}$ & $1.2 \times 10^{-4}$ \\
Radiative multicomponent ...... & $1.68 \times 10^{16}$ & 120 & 190 & $1.0 \times 10^{4}$ & $6.5 \times 10^{-5}$ \\
\hline
\end{tabular}

Note.- Values given are maximums in jet. 
jet core and collar. When the equilibrium jet encounters the external medium, the various elements of the equilibrium are shocked. This creates two bow shocks and a cocoon. The bow shock closest to the axis takes the form of a nose cone. Intrinsic instabilities develop in the inner part of the shocked core, as well as in the cocoon. The annotated Figure 3 sums up this section by showing the set of common features on a characteristic multicomponent jet. We now focus on the propagation characteristics of the two types of input equilibria.

\subsection{Keplerian Rotator}

To briefly review Keplerian rotators produce jets with a nearly constant velocity cross section. The mass density is stratified with a high-density core surrounded by a lower density collar. The core-collar density ratio for the present jet is relatively low: $\rho_{\text {core }} / \rho_{\text {col }}=1.67$, (this is also the density ratio between the core and the ambient medium, $\eta=\rho_{j} / \rho_{a}$ ). The toroidal magnetic field in the jet reaches its maximum value just at the outer edge of the core. As we shall see, the coupling of higher density in the core with the strong magnetic stresses along the core/collar boundary dominates the propagation characteristics of the entire jet.

\subsubsection{Keplerian Rotator: Adiabatic Jet}

3.2.1.1. Propagation

In Figure 4 we present gray-scale maps of the density evolution of an adiabatic jet driven by a Keplerian rotator. In the first frame, taken at $t=5.3 \tau$, the classic jet shock/bow shock pair are apparent. The bow shock accelerates the ambient gas, while the jet shock decelerates the jet material. The speed of the jet head or bow shock is $v_{h} \approx 77 \mathrm{~km} \mathrm{~s}^{-1}$. This speed is relatively constant throughout the simulations. Frank et al. (1998) derived a formula for the bow shock speed that accounted for magnetic pressure in the beam. Using the familiar result for hydrodynamic jets, $v_{h o}=v_{j} /(1+1 / \sqrt{\eta v}),(v$ is the ratio of bow shock and jet head radii $\left.R_{h} / R_{j}\right)$ the MHD bow shock speed is.

$$
v_{h}=v_{h o} \frac{1-\sqrt{1 / \eta v-p^{*} / \rho_{j} v_{j}^{2}(1-1 / \eta v)}}{1-(1 / \sqrt{\eta v})},
$$

If we take $v=1$ then this equation gives $v_{h} \approx 70 \mathrm{~km} \mathrm{~s}^{-1}$ with magnetic pressure accounting for approximately $4 \%$ of the momentum flux driving the shock. As was noted in Frank et al. (1998), the higher velocity of the jet head seen the simulations can be attributed to the aerodynamic effect of streamlining the jet head via MHD hoop stresses (an $v$ effect). The nose cone shape that develops reduces the drag on the jet head increasing its velocity relative to a more blunt jet head that would occur in a pure hydrodynamic simulation.

In the first frame of Figure 4 we already see the effect of the core/collar structure on the jet propagation. The higher density core, confined by the magnetic hoop stresses, maintains its structural integrity on the downstream side of the jet shock. It is noteworthy at this early time that the core appears to propagate ahead of the rest of the beam. This is to be expected purely from momentum considerations as equation (12) predicts a $\Delta v=10 \mathrm{~km} \mathrm{~s}^{-1}$ difference in the speed of the core and collar bow shocks. Detailed examination of the simulations also shows that at these early times there is little material flowing from the jet into the cocoon. This is apparent in Figure 5, which shows the poloidal plane velocity vectors for the head of the jet. The origin of this effect lies, once again, in the relative strength of the toroidal field in the core and collar. What material does flow into the cocoon comes primarily from the outer most regions of the collar $\left(r>.75 R_{j}\right)$. Note that there is no transverse motion in the shocked core material. We attribute this to magnetic forces. In the region where material is flowing in the radial direction the magnetic field has an average value that is $\frac{1}{3}$ that at the core/collar interface. A better means of judging the relative strength of the field comes

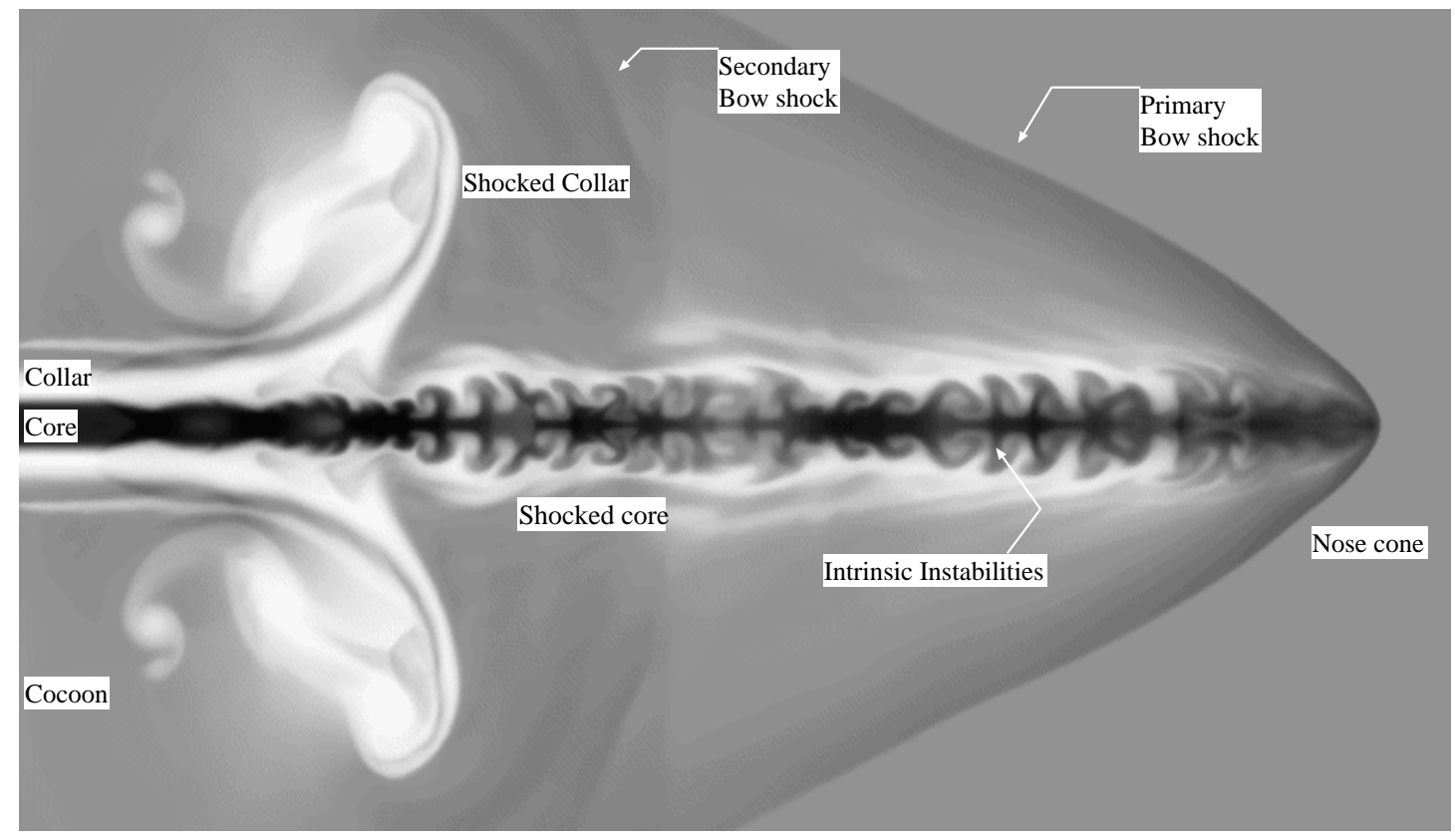

FIG. 3.-Annotated gray-scale map of the density showing the basic features that are common to most of the simulations. This corresponds to the adiabatic simulation of the multicomponent jet at time $t=8.6$. 


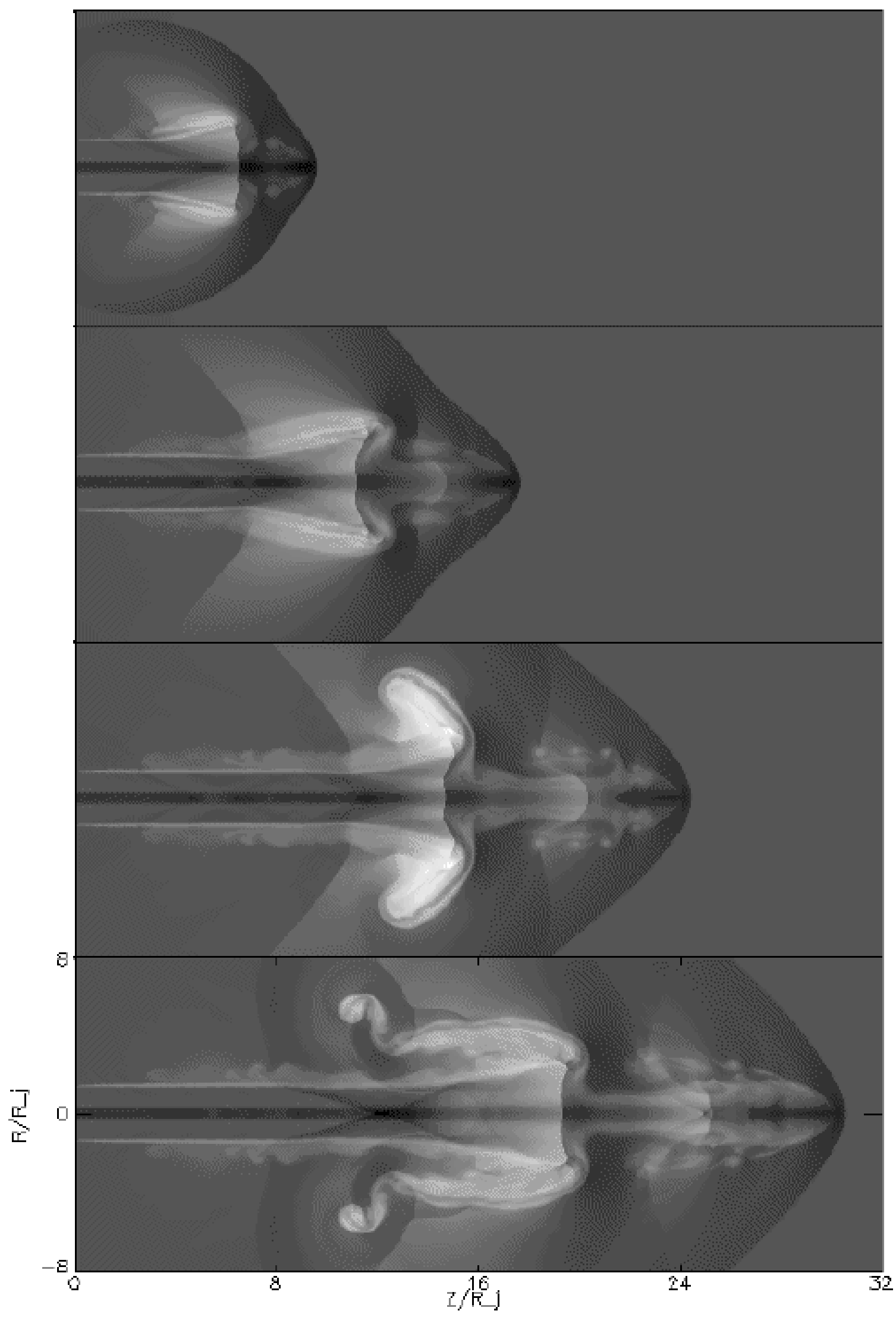

Fig. 4. - Gray-scale maps of density for adiabatic simulations of Keplerian jet. Four frames from the simulation are shown. From top to bottom the times are $t=5.3,10.6,15.9,20.2$. The horizontal $(Z)$ and vertical $(2 R)$ dimensions of the simulation are $Z=32 R_{j}$ and $R=8 R_{j}$, respectively. 


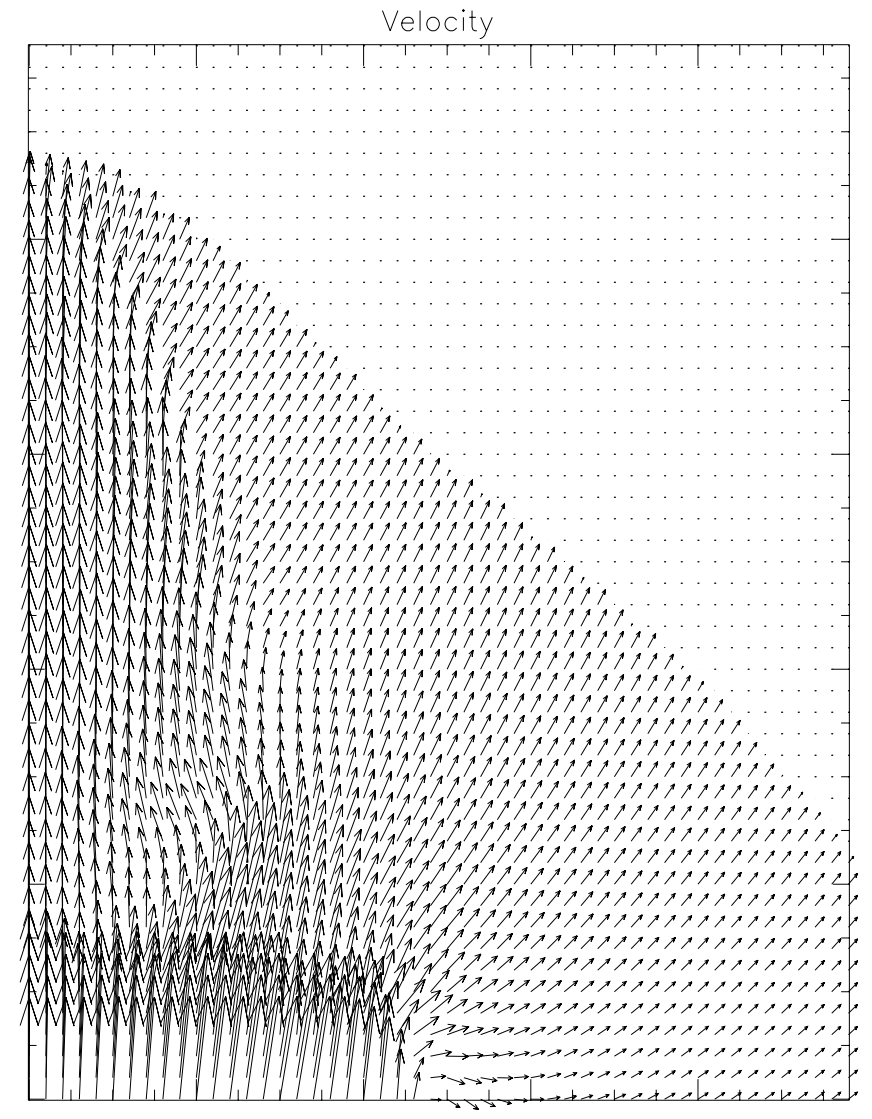

FIG. 5.-Poloidal velocity vectors at the head of adiabatic Keplerian jet. The longest vectors correspond to a speed of $v=99 \mathrm{~km} \mathrm{~s}^{-1}$. The figure is taken at $t=5.3$ and the size is $3.8 R_{j}$ on each side.

from examination of the plasma parameter $\beta$. Downstream of the jet shock the core collar interface has $\beta=3$, while the collar/ambient interface has $\beta=23$. Thus fields can exert stronger stresses along the core restricting its lateral expansion.

The remaining frames of Figure 4 show the distance between the jet and bow shock continues to grow. Note that as the jet evolves the core never loses its identity. It acts, essentially, as a jet within a jet. At later times we see secondary shocks developing within the shocked core as well as vortex shedding at the head of the jet at radii consistent with the core/collar interface. In particular, by the second frame we see what appears to be a second jet shock forming inside the core as it pushes through the ambient gas. Note also that at later times the shocked core material takes on the familiar nose cone morphology seen in top-hat MHD jets with strong toroidal geometries. These features emphasize the apparent independence of the core's propagation characteristics relative to the rest of the jet. Thus our results show that the core/collar dichotomy appears to control the main features of the jet beam via gradients in inertia and Maxwell stresses.

\subsubsection{The Lateral Expansion}

The second notable feature in the adiabatic Keplerian jet simulations is the large-scale mass expulsion event that occurs in the third frame in Figure 4. The plasma driven laterally (in the $r$ direction) in this event is composed entirely of shocked collar material. At early times the cocoon is fed solely through the outer annuli of the jet as Figure 5 demonstrated. The hoop stresses in the material in the collar at smaller radii are, however, too strong to allow plasma to stream transversely into the cocoon. To see this explicitly consider the radial momentum equation where terms involving $v_{\phi}, B_{r}$ are ignored and we also ignore variations in $z$.

$$
\frac{\partial \rho v_{r}}{\partial t}+\frac{1}{r} \frac{\partial}{\partial r}\left(r \rho v_{r}^{2}\right)=-\frac{\partial}{\partial r}(p)-\frac{\partial}{\partial r}\left(\frac{1}{2} B_{z}^{2}+B_{\phi}^{2}\right)-\frac{\left(B_{\phi}^{2}\right)}{r},
$$

The last term on the right is the hoop stress. The second to last term is the magnetic pressure. From Figure 2 it is clear that for $r>.3 R_{j}$ both the gas and magnetic pressure gradients are negative. Thus in these regions the hoop stress opposes the pressure forces and acts to constrain lateral expansion of the flow.

At later times, however, two features occur that alter the balance of forces. First as more material builds up immediately behind jet shock, both the gas pressure and magnetic pressure increase relative to the magnetic tension. Second, and most importantly, the jet shock becomes distorted, tipping toward direction of jet propagation. The jet shock becomes conical with the vertex of the cone pointing into the undisturbed beam. Thus the jet shock becomes oblique relative to the unshocked mass flux. The shock conditions for velocity lead to the following expression for the postshock radial velocity

$$
v_{r} \approx \frac{1}{4} v_{j} \cos (\theta) \sin (\theta),
$$

where $\theta$ is the angle between the jet shock and the $z$-axis and $v_{j}$ is the jet velocity relative to the jet shock. For $\theta<90^{\circ}$ the postshock gas acquires a significant $v_{r}$ component. Material in the beam is refracted away from the axis. The momentum flux in the outward radial ram pressure, $\rho v_{r}^{2}$, is able to overwhelm the magnetic tension force leading to a large-scale expulsion of material into the cocoon.

\subsubsection{The Shocks}

It is difficult to isolate the processes that cause the bending of the jet shock into a conical shape. The dynamics at the jet head are highly nonlinear and time-dependent and it is not obvious if the change in shock geometry is an amplification of events downstream where the flow pattern is quite complicated or if the distortion can be linked to events upstream. Close inspection of the simulations gives the impression that the distortion of the jet shock occurs after a pinch wave reflects off the axis just upstream of the jet shock in the unshocked beam. The origin of the pinch appears to come from the slow expulsion of material into the cocoon prior to the third frame. In the early evolution the cocoon distorts the flow of ambient gas behind the bow shock, i.e., the cocoon represents an obstacle that the postbow shock flow must stream around. As the shocked ambient material streams over the cocoon it becomes transonic. Its return to parallel streaming along the jet boundary can only occur via an additional shock. This feature is apparent at $z \approx 8 R_{j}$ in frame 2 of Figure 4. Such flow patterns are well known to aerodynamicists as they are common in aerofoil theory (Ramm 1990). It appears that the pinch wave is generated just downstream of this addi- 
tional shock and may be attributable to the higher pressures generated on the surface of the jet.

After the large mass-expulsion event the jet shock appears to relax to a configuration where it is perpendicular to the $z$-axis $\left(\theta=90^{\circ}\right)$ and the flow into the cocoon is reduced. As the expelled material curls back toward the jet beam, however, it impinges on the jet surface and another, strong pinch is generated. At the end of the simulation (after the jet head has moved off the grid) we find the jet shock becoming distorted yet again leading, perhaps, to a second mass-shedding event.

\subsubsection{The Magnetic Effects}

In Figure 6 a we show the magnetic field structure in the adiabatic Keplerian jet. The figure shows the poloidal $\left(\boldsymbol{B}_{p}=B_{r} \hat{e}_{r}+B_{z} \hat{e}_{z}\right)$ magnetic field lines and the toroidal field $\left(B_{\phi}\right)$. The field clearly traces out the main features of the flow described above: the nose cone at the jet head; the mass ejected behind the jet shock; the pinch wave occurring where the ejected mass is swept back onto the jet beam. It is noteworthy that it is the toroidal field that articulates these structures most clearly. This is appropriate as the toroidal field dominates in the jet providing much of the force that shapes the jet dynamics. Note that the figure indicates that the magnetic pitch $B_{\phi} /\left|B_{p}\right|$ increases behind shocks. This is a general feature of helical fields in jets. In a fast MHD shock only the component of the field parallel to the shock face is strengthened via compression (the parallel component will scale as $\rho$ ). Thus in a jet with a helical magnetic topology shock waves act to comb out the field leading to enhanced toroidal fields (and hoop stresses) in the postshock regions (Gardiner \& Frank 2000). We note however that shear in the flow will also lead to significant strengthening of the field via stretching of field lines.

Our results for the Keplerian model show that the behavior of a jet with a more realistic initial density, pressure, and magnetic field structure leads to propagation characteristics that have not been seen in previous hydrodynamic jet simu- lations. As we shall see this theme is repeated in all the simulations.

\subsubsection{Keplerian Rotator: Isothermal/Radiative Jet 3.2.2.1. The Propagation}

In Figure 7 we present gray-scale maps for the density evolution of the radiative Keplerian rotator jet. Recall that the radiative model begins with modified initial conditions compared with the adiabatic or isothermal Keplerian simulation. In order to keep the initial temperature at $T=T_{o} \approx$ $10^{4} \mathrm{~K}$, we scaled down all the radial distributions of all variables in the jet except $v_{z}$. This had the additional effect of producing a jet with a higher Mach number, $M_{f} \approx 7$. We note that the basic features seen in the radiative simulation are quite similar to those seen in the isothermal model $(\gamma=1.001)$. Thus for brevity we do not present the isothermal results. The only notable difference between the two models is the width of the bow shock. This can be understood purely in terms of the opening angle $\theta_{c}$ of the Mach cone for a supersonic flow, $\theta_{c}=\sin ^{-1}\left(1 / M_{f}\right)$. We see a wider opening angle for the lower Mach number isothermal flow as expected from the relation for $\theta_{c}$.

The dynamics of both the isothermal and radiative simulations are dominated by the loss of pressure support between the jet- and bow shocks. In the isothermal model this occurs because $P \propto T_{o}$ where $T_{o}$ is a constant equal to the ambient temperature. In the radiative model the gas behind both the bow- and jet shocks are driven to temperatures of $T=(3 / 16)(\mu / k) V_{s}^{2} \approx 10^{5} \mathrm{~K}$ where $(s)$ refers to the shock speeds. The cooling time for a jet with $n_{j}=100$ $\mathrm{cm}^{-3}$ is $t_{c}=.25 T /\left(n_{j} \Lambda(T)\right) \approx .2(\tau)=5 \mathrm{yr}$. Thus we expect the postshock gas at the head of the jet to cool effectively and for the dynamics to be, essentially isothermal. This is confirmed by consideration of the first frame in Figure 7, which shows by $t=1.38$ the two shocks have already collapsed on to each other producing a thin shell. The densities and magnetic field strengths in the shell are high, with 1000 $\mathrm{cm}^{-3}<n<6000 \mathrm{~cm}^{-3}$ and $100 \mu \mathrm{G}<B<300 \mu \mathrm{G}$. Unlike purely hydrodynamic radiative shocks, MHD radi-

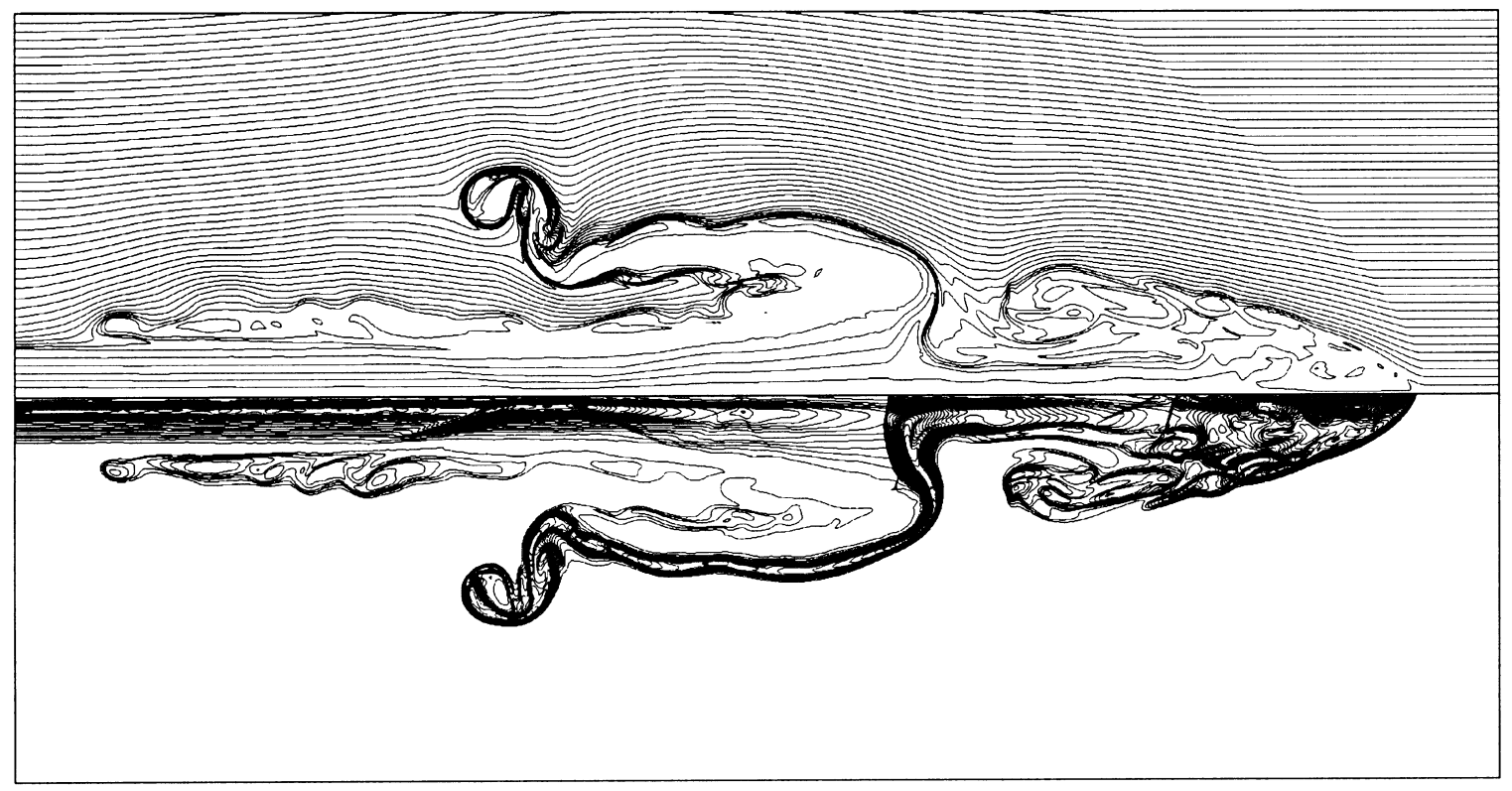

FIG. 6.-Magnetic field for Keplerian jet. Adiabatic simulation at $t=20.2$. The top half contour plot represents the poloidal field $\left(\boldsymbol{B}_{p}=B_{r} \hat{e}_{r}+B_{z} \hat{e}_{z}\right)$. The bottom half contour plot represents the toroidal field $\left(B_{\phi}\right)$. Note the increase in the magnetic pitch $B_{\phi} /\left|B_{p}\right|$ behind shocks. 


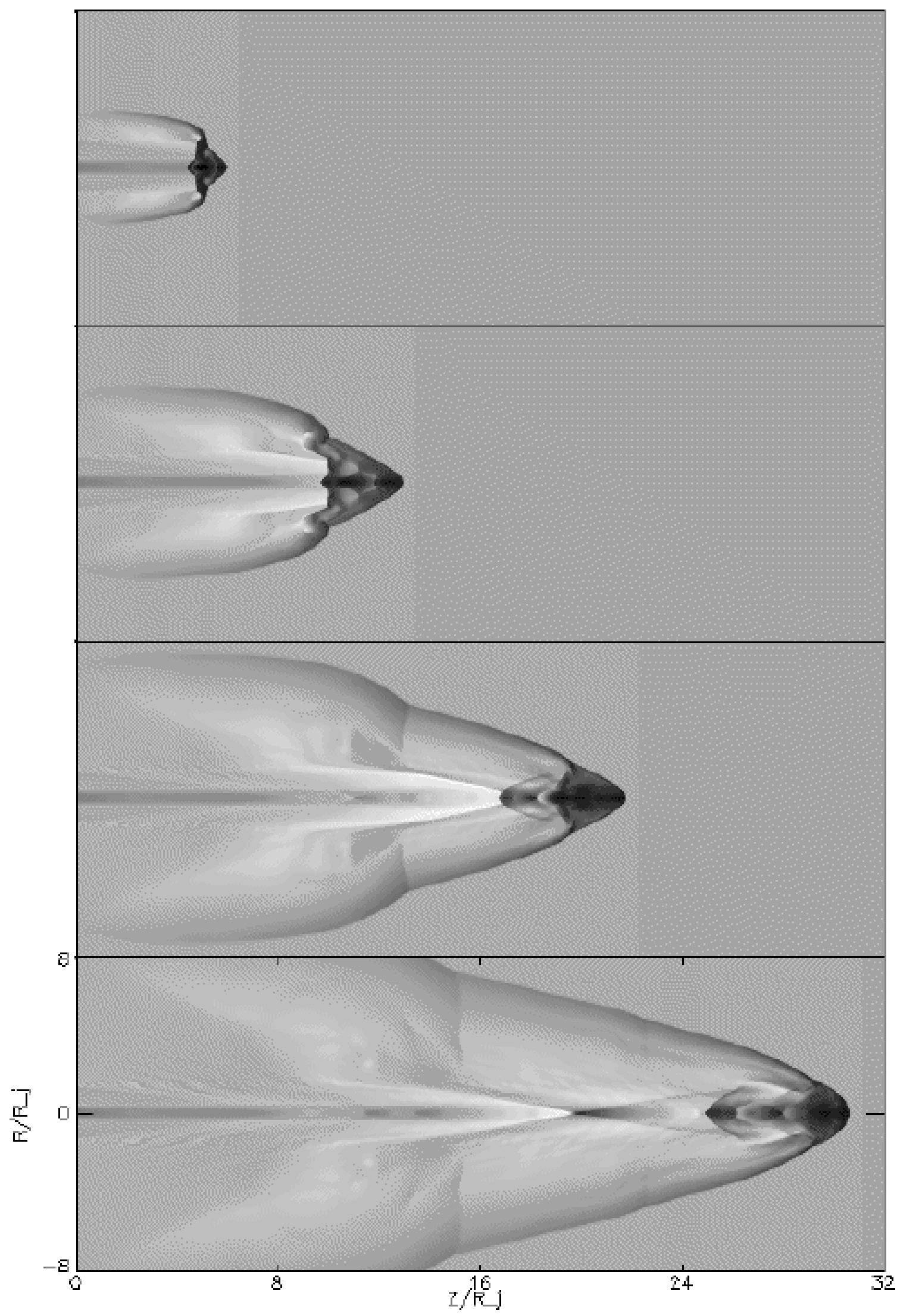

Fig. 7.-Gray-scale maps of density for radiative simulations of Keplerian jet. Four frames from the simulation are shown. From top to bottom the times are $t=1.6,3.5,5.9,8.6$. 
ative shocks possess a theoretical limit for the postshock compression. This occurs because of magnetic pressure exerted by the component of the field perpendicular to the shock normal. Equating ram pressure and magnetic pressure allows a simple form of the maximum postshock density to be derived (Hollenbach \& McKee 1979),

$$
n_{m}=\frac{\left(2 m_{h}\right)^{1 / 2}\left(n_{j}\right)^{3 / 2} v_{s}}{B_{\phi 0}} .
$$

Note the above use our scaling for the field. For the Keplerian jet $n_{m} \approx 1000 \mathrm{~cm}^{-3}$, which is in good agreement with the simple prediction above. The higher densities achieved in the simulation come from the pinch force induced compression on the axis

3.2.2.2. The Shocks

The effect of the radial stratification in both the density and magnetic field (the core/collar structure) are already apparent at the first frame of the simulation just as in the adiabatic models. In the radiative simulation however, the bow shock quickly assumes a pointed, cusplike shape. This is due to the higher density in the jet core and the magnetic pinch forces from the strong toroidal field. Compared with the adiabatic simulations described above the head of the jet assumes what might described as a "bullet" shape rather than a nose cone. In Frank et al. (1998) significant streamlining was observed in the radiative simulations compared with adiabatic ones. This was attributed to the loss of thermal energy and, hence, the increased effectiveness of magnetic stresses. Here we see a similar effect, which is enhanced by the increased ram pressure in the jet core relative to the collar.

We also see a mass-shedding event in these simulations though it is far weaker than what occurs in the adiabatic models. Frame 2 of Figure 7 shows the initialization of the event. As in the adiabatic models a secondary bow shock wave generated by his event leads to pinching of the jet beam and a downstream distortion of the jet shock.

Note the cusp that appears in the jet shock in the first frame of the simulation. While all features on the axis of an axisymmetric simulation must be taken with some suspicion, a close examination of the simulation data reveal a straightforward explanation for this structure. The strongest postshock field values in the jet head occur just downstream of the jet shock at a radius where the preshock field is a maximum. Recall that this occurs just on the outside edge of the jet core $r \approx 3 R_{j}$. This is also where $\beta$ drops to its lowest value, $\beta \approx .5$. Thus magnetic stresses dominate the plasma at this location in the jet head. At radial positions just inward of the point where $\beta=\beta_{\min }$ we find $v_{r}$ obtaining is maximum inward (negative) value. This radially inward flow is apparent in Figure 8, in which we present the velocity vectors at the head of the jet. Thus, at positions immediately downstream of the jet shock magnetic forces squeeze and compress the jet core. Given the strong cooling, the relation $P \propto \rho$ is approximately valid and the axial location of the pinch is a local pressure maximum. The downstream pinch can communicate upstream with the jet shock face, thus producing the bulge or cusp that faces into the oncoming material in the beam. Note that this feature was not seen in the adiabatic models because there the postshock gas pressure was high enough to inhibit the strong pinch. In fact, $\beta$ will always increase across an adiabatic shock. If we write the postshock compression as $X=\rho_{2} / \rho_{1}$

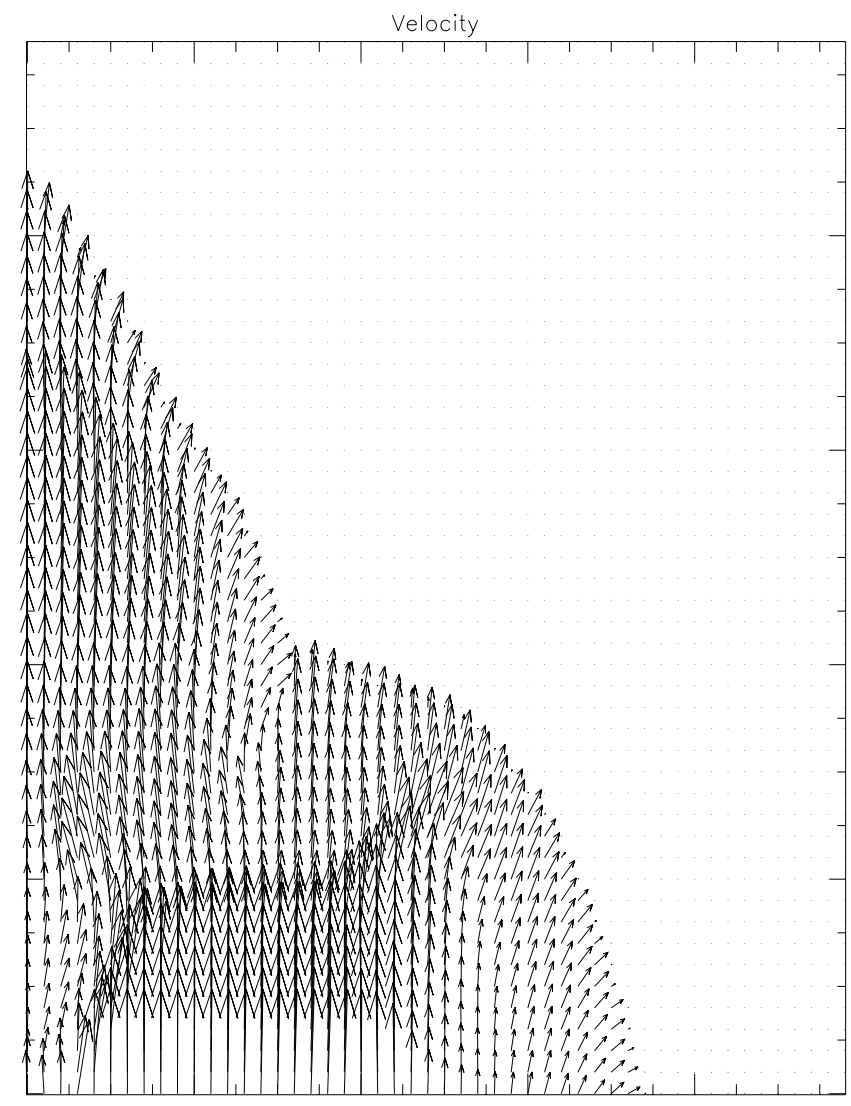

FIG. 8.-Poloidal velocity vectors at the head of radiative Keplerian jet. The longest vectors correspond to a speed of $v=120 \mathrm{~km} \mathrm{~s}^{-1}$. Note that the largest radially inward directed flow occurs where $\beta=\beta_{\min }$. The figure is taken at $t=5.9$ and the physical size is $3.8 R_{j}$ in vertical direction.

with the subscripts 1 and 2 corresponding to pre-and postshock conditions, respectively, then (Priest 1986)

$$
\frac{\beta_{2}}{\beta_{1}}=\gamma M_{f}^{2}\left(1-\frac{1}{X^{3}}\right)-\frac{1}{\beta_{1}}\left(1-\frac{1}{X^{2}}\right) .
$$

The equation above shows that for strong adiabatic shocks $\left(M_{f} \gg 1, X \approx 4\right) \beta_{2}>\beta_{1}$. It is only when the postshock thermal energy is lost to radiation that the postshock magnetic forces can dominate.

The magnetic field structure in the jet is shown in the bottom frame of Figure 9. The most prominent feature of the field configuration is the compact size and high field strengths in the jet head. The effect of the pinch wave is clearly apparent upstream of the jet head. The field also shows the effect of the weaker mass-shedding event that occurs in this model. Note the isolated loops of $B_{\phi}$ and strong distortion of $B_{\mathrm{pol}}$ behind the cusp in the bow shock. It is also notable that the turbulence and multiple instabilities that are seen in most radiative jet simulations do not occur here. As Gardiner \& Frank (2000) have found for their pure poloidal simulations this is one of the principle effects of strong magnetic fields. Thus if YSO jets do contain strong embedded fields then one must consider what their effect on the morphology of the HH objects should be.

\subsection{Multicomponent Rotator}

The structural differences between the Keplerian and multicomponent jet originate primarily in the differences in density and velocity cross sections. As we saw in Figure 2 


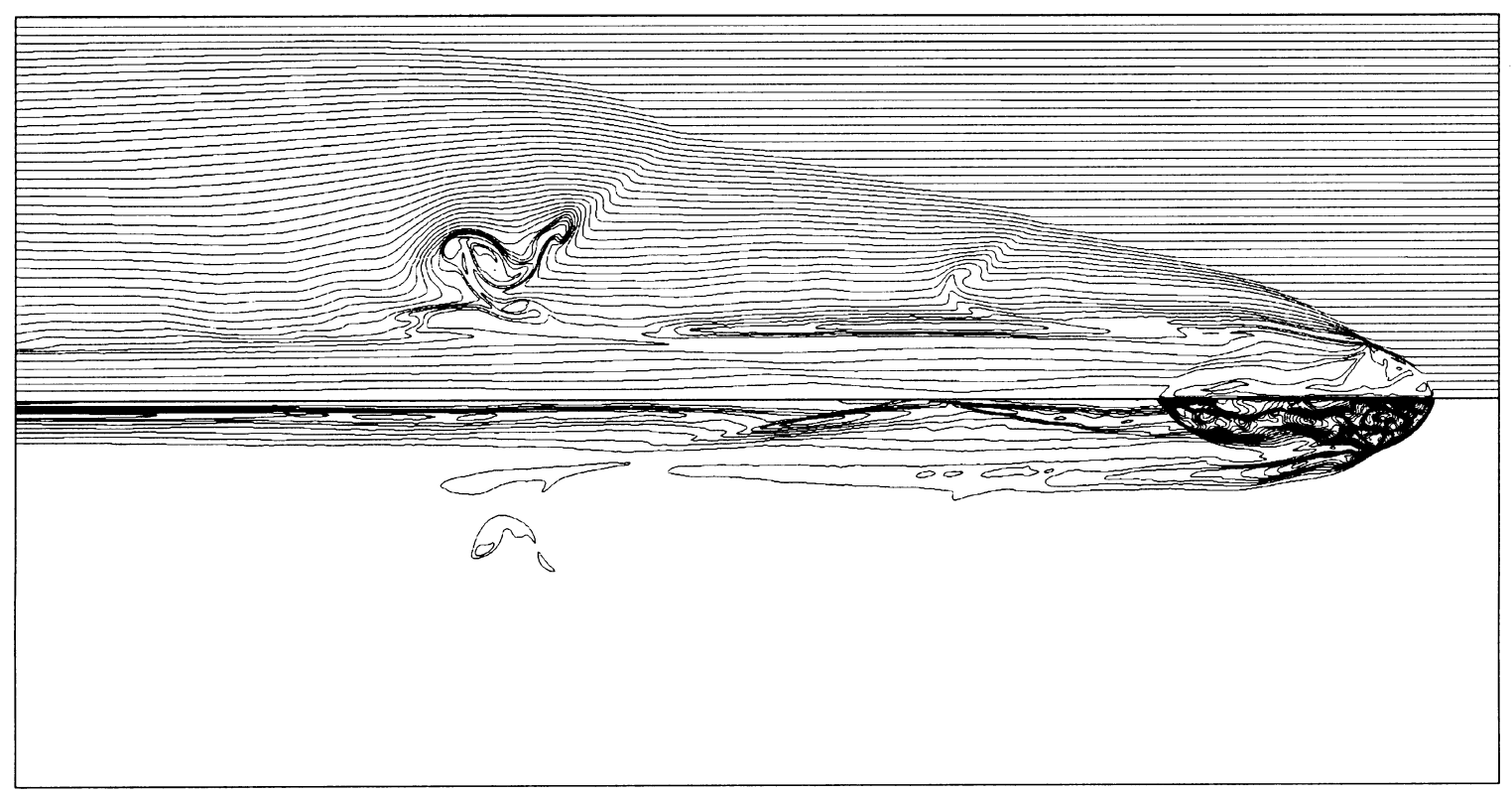

FIG. 9.- Magnetic field for Keplerian Jet. Radiative simulation at $t=8.6$. The top half contour plot represents the poloidal field $\left(\boldsymbol{B}_{p}=B_{r} \hat{e}_{r}+B_{z} \hat{e}_{z}\right)$. The bottom half contour plot represents the toroidal field $\left(B_{\phi}\right)$. Note the increase in the magnetic pitch $B_{\phi} /\left|B_{p}\right|$ behind shocks.

the multicomponent jet has three structural elements: a high-density core; a low density inner collar; a moderate density outer collar. The ratio of the peak density in the core to minimum density in the inner collar is $\rho_{\max } / \rho_{\min }=$ 100. This is almost 2 orders of magnitude higher from what is obtained in the Keplerian jet. In addition, the velocity in the jet peaks in the inner core just at the point where the density drops with $V_{\max } / V_{\min }=1.7$. In the last section we discussed how the (milder) cross-sectional variations in the Keplerian jet had important dynamical consequences for its propagation characteristics. Thus we expect the more extreme radial variations in the multicomponent jet will likely effect the dynamics in more extreme ways.

\subsubsection{Multicomponent Rotator: Adiabatic Jet} 3.3.1.1. Propagation and "Peel-off"

Figure 10 shows the evolution of the multicomponent jet through four gray-scale maps of log density taken at different times in the evolution of the simulation. The most prominent feature in the flow is what we have termed the "peel-off" of the jet's outer collar. As the jet propagates down the grid, the outer collar develops a strong radial velocity component. As the outer collar expands sideways it is decelerated and develops into a large-scale vortex. This is somewhat similar to what was seen in the mass-expulsion event seen in the Keplerian case. The high-density core of the jet continues its forward propagation driving through the ambient medium at high speed and quickly pulls away from the decelerated collar. By the end of the simulation the "naked" core has propagated far downstream where it encounters the ambient medium in a manner unaffected by the outer collar.

The origin of the peel-off appears to reside in the density stratification of the jet. First we note that since the peel-off occurs early in the simulation we must be suspicious of it as an artifact of the way the simulations are initiated. Given the nature of this study it is difficult to circumvent the need to begin our simulations with a fully formed jet as this is the point of the project. As was noted in $\S 2$, experiments in which the smoothing length between the jet and ambient conditions was varied revealed no change in the propagation characteristics. Even when the initial head of the jet was smoothly joined with ambient medium over a length of many jet radii we found the development of the peel-off was only delayed. The outer layers always developed their transverse motion and the evolution was identical to models with shorter or no smoothing transition. Thus, while the development of this feature may be a transient, it is a highly robust one. Consideration of the dynamics inherent to stratified jets such as these however allows one to infer the mechanism driving the peel-off. We focus on the jet shock. The highest postshock pressures occur behind the highest velocity regions of the jet. This occurs in the low density inner collar. Since $\eta(r)<1$ in this region the jet shock is relatively strong and is pushed back into the jet deeper than in either the core or outer collar. The oblique geometry of the inner shock generates a strong transverse flow in both positive and negative radial directions. This can be seen in Figure 11, which shows the poloidal flow vectors. In addition, the variation of the inner shock produces a finger of high-pressure gas, which reaches back into the jet in the low density inner collar. A strong radial pressure gradient is established, which drives the outer collar away from the core much like splitting wood with an axe. Once the sideways expansion begins, the ram pressure of the ambient medium (in the frame of the jet) continues to divert the flow of the outer collar.

The difference in jet propagation speeds between the multicomponent and Keplerian jets is also dramatic. The velocity of the bow shock at the end of the simulation is $V \approx 100 \mathrm{~km} \mathrm{~s}^{-1}$, which is a $25 \%$ increase over the propagation speed of the Keplerian jet. This difference can be attributed two effects. First, the multicomponent jet has a higher value of $\eta$ in the core relative to the ambient medium ( $\eta \approx 6.8$ for the multicomponent jet). From equation (12) this translates into a relative propagation velocity difference of $14 \%$. The excess in propagation speeds above this is likely to be attributable to a second effect - the streamlining 


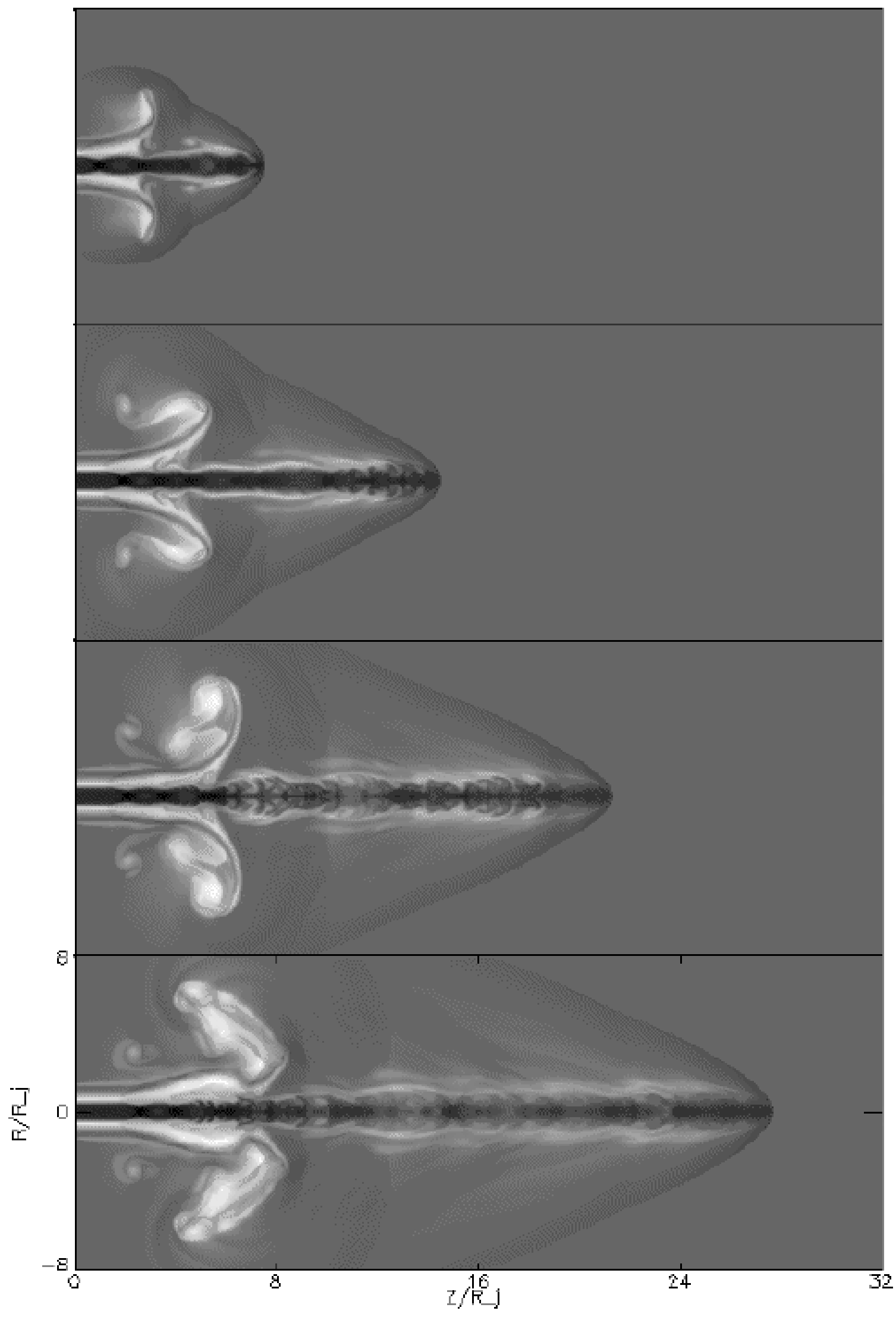

FIG. 10. - Gray-scale maps of density for adiabatic simulations of multicomponent jet. Four frames from the simulation are shown. From top to bottom the times are $t=2.9,5.7,8.6,11.4$. The horizontal $(Z)$ and vertical $(2 R)$ dimensions of the simulation are $Z=32 R_{j}$ and $R=8 R_{j}$, respectively. 


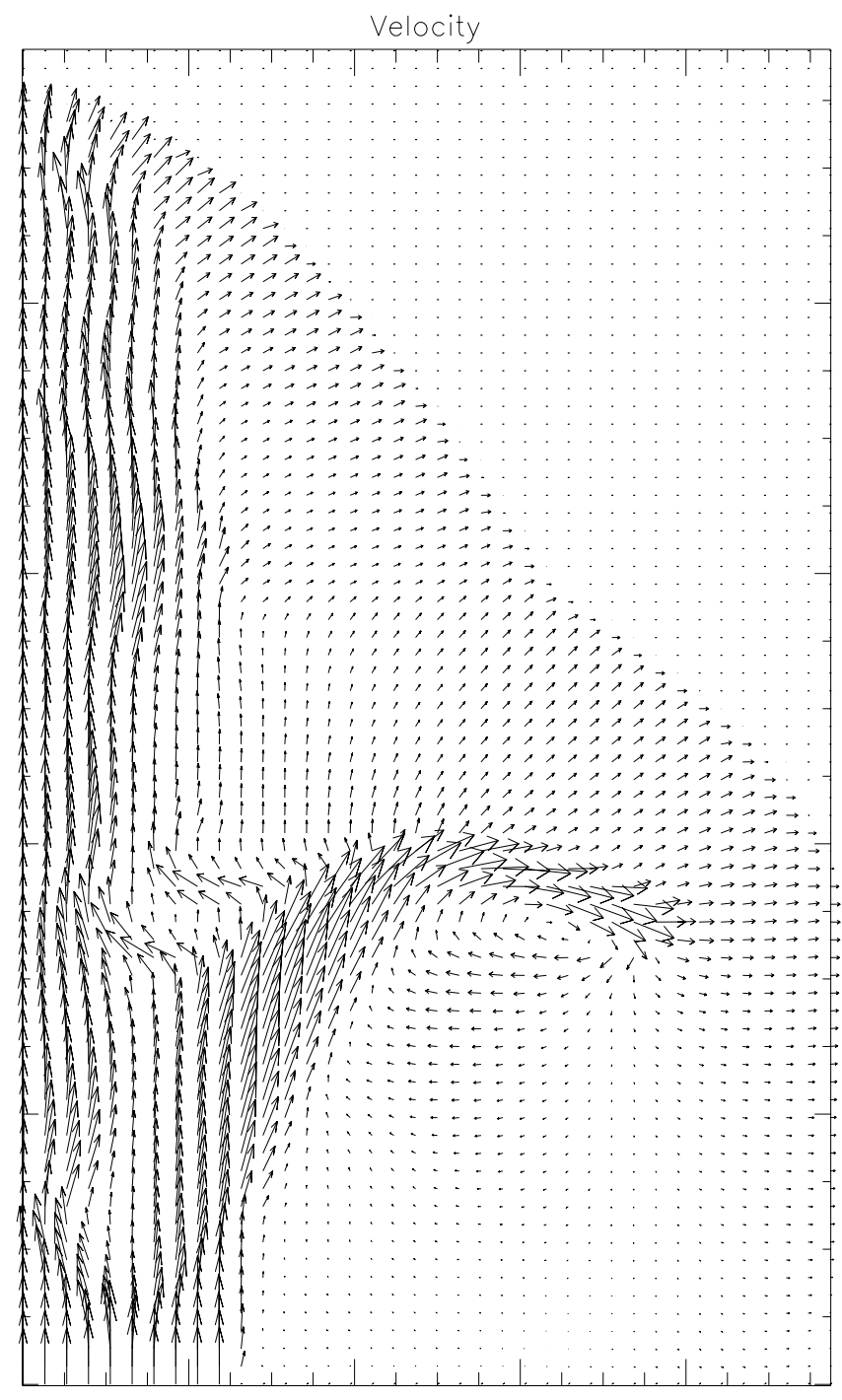

FIG. 11.-Poloidal velocity vectors at the head of adiabatic multicomponent jet. The figure is taken at $t=2.9 \mathrm{yr}$ and the physical size is $4.5 R_{j}$ in vertical direction. The longest vectors correspond to a speed of $v=120 \mathrm{~km} \mathrm{~s}^{-1}$.

of the jet head. Once the outer collar peels away from the core, the jet presents a smaller and more streamlined head to the ambient medium allowing it to propagate at higher speeds. This can be seen by comparing the bow shock opening angles for the multicomponent and Keplerian jets. Note the streamlining of the head of the naked core also comes via the strong magnetic pinch forces at its outer radial edge and at late times the core also develops the familiar nose cone morphology.

\subsubsection{The Instabilities}

The development of strong instabilities in the core of the multicomponent jet is another notable characteristic of the simulations. Once the core is exposed we see periodic pinches in the beam. As the instabilities evolve they expand radially and develop a arclike shape. At later times these arcs become swept backward by shear in the beam. Further evolution leads to a loss of their sharp edges and individual arcs begin to merge. From detailed consideration of animations of the simulations it appears that the instabilities first appear near the head of the naked core. Only at later times as the peel-off the outer layers continues do they appear immediately downstream of the peel-off region. This is most likely an indication of where the perturbations driving the instabilities occur.

We have performed a stability analysis of the multicomponent magnetic configuration (Lery 1996; Lery \& Frank 2000). A global normal mode stability analysis was performed using the same method as in Appl, Lery, \& Baty (2000). In these two papers the stability of magnetized astrophysical jets with respect to modes driven by the electric current density distribution was addressed. The results show that the current driven (CD) instabilities grow rapidly on timescales of order of the Alfvén crossing time in the jet frame and that they are likely to modify the magnetic structure of the jet. Since they are internal modes (see Appl et al. 2000) the CD instabilities should not disrupt the jet. In the present work, we have focused on the pinch mode because of the axisymmetric nature of our calculations. In three dimensions it is likely that the kink mode may play a role as well but should not break the integrity of the jet. This is because the jet is superfast and should see its boundary as a rigid wall. Consequently, the instabilities should be mainly internal, the jet would not be disrupted. The instabilities should certainly be expected to change the jets magnetic configuration drastically.

The analysis shows that the strong pinch (or sausage) mode is mainly due to large gradients of the density and magnetic field. In Figure 12, we have plotted the dispersion relation for different values of the external pressure surrounding the jet. We have adopted the standard temporal approach where the axial wavenumber is real and the imaginary part of the complex frequency corresponds to growth rate. Wavenumbers are given in units of inverse jet radius and growth rate is normalized to the inverse Alfvén time. It has been found that the location of the peak mainly depends on the magnetic distribution in the jet. The short- $k$ cutoff is due to the finite size of the jet radius that has an external boundary that behaves as a rigid wall for Mach numbers larger than unity (see Appl et al. 2000). We find that pure magnetic instabilities driven by electric current

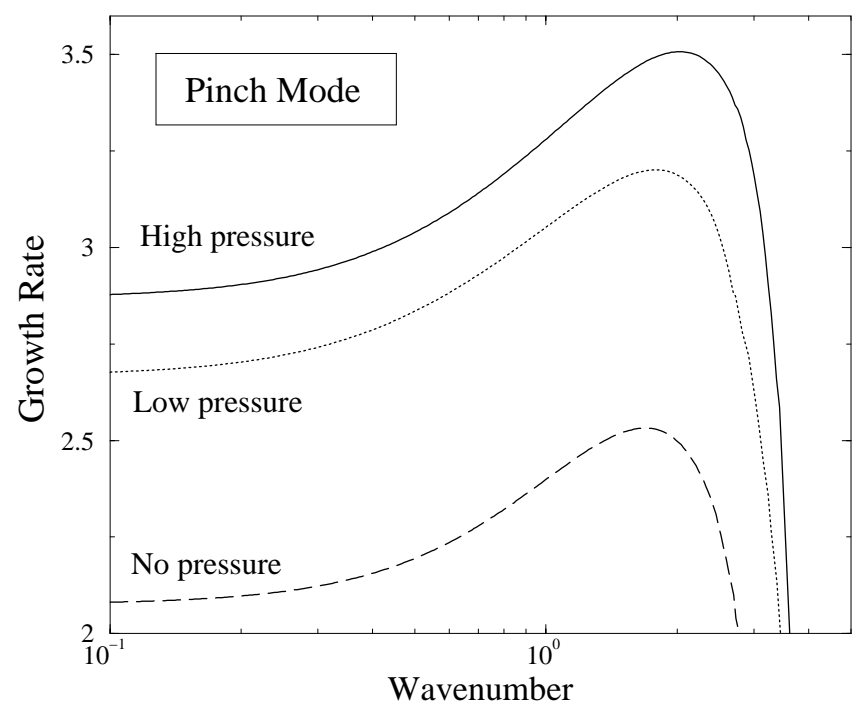

FIG. 12.-Stability Analysis. Dispersion relation, i.e., growth rate, vs. wavenumber, for the pinch mode with different values of the external pressure surrounding a multicomponent jet. 
develop on rapid Alfvén timescales. Also, Figure 12 clearly shows that when the external pressure increases the jet becomes more unstable. This is precisely what we observe when the core becomes naked downstream of the peel-off region. It also explains why the instabilities do not develop as rapidly for the Keplerian case where the density and pressure gradients are less important.

From the simulations it appears that at later times waves driven off the peel-off region seed the instabilities, while at earlier times the seeds occur via shocks at the jet head. As can be seen in Figure 10 the pinching instabilities on the axis have a wavelength of approximately $\lambda \approx .5 R_{j}$. Note, however, the presence of a second characteristic wavelength, which runs along the surface of the core. This feature, which appears as an envelope encompassing the shorter wavelength modes, has $\lambda \approx 3 R_{j}$.

The stability analysis of multicomponent jets shows that the unstable modes that should grow the most rapidly have a wavelength of about 3 jet radii for the collar and half the jet radius for the core. These results have been reported by Lery \& Frank (2000). They are in good agreement with the simulations, and can be also compared to observations. For example, the jet of HH34 presents a mean knot separation of 3.4r $r_{\mathrm{jet}}$ as given by Burke et al. (1988). Thus, the present results suggest that these instabilities could be at the origin of the knotty structure of a large number of jets as seen, for example, in HL Tau, HH1, HH30, and HH34 (Ray et al. 1996).

Finally consider the magnetic structure in the jet, which is shown in Figure 13. The field structure is quite complicated as might be expected. Note the form of the bow shock in the $B_{\text {pol }}$ component as well as its the relative absence in the peeled off outer collar, which is dominated by toroidal fields. Examination of $\beta$ in these regions shows that the gas remains hydrodynamically dominated with $\beta \gg 1$ in spite of amplification from both the shocks and radial stretching. Within the core the pinch modes are clearly seen in the toroidal component with specific islands in the beam corresponding to regions of strong pinch. Numerous islands of
$B_{\mathrm{pol}}$ are created by the instabilities indicating the presence of reconnection.

\subsubsection{Multicomponent Rotator: Isothermal/Radiative Jet}

In Figure 14 we present gray-scale maps of the density evolution of a radiative jet driven by a multicomponent rotator. The radial distributions were scaled down for all variables except $v_{z}$ in the jet such that $M_{f} \approx 9$. The basic features of the simulation are similar to the isothermal model however there are some differences. To address these we also present in Figure 16 a single frame from the isothermal simulation. Note first that, once again, the width of the bow shock is reduced in both the radiative and isothermal case relative to adiabatic model. This can be attributed both to cooling and the increase in fast mode Mach number.

The most important point to notice in this simulation is that the outer collar still peels away from the jet core, which then propagates ahead of the rest of the flow. This occurs even though the cooling is strong. As in the adiabatic model, the initiation of transverse flow in the outer layers occurs owing to the shape of the jet shock. As the first frame of Figure 14 demonstrates, with cooling included both the bow shock and jet shock effectively "drape" around the head of the jet. This feature occurs as a result of the loss of pressure support behind the shocks. As in the adiabatic model the peel-off appears to be primarily driven by the redirection of the flow behind the oblique jet shock. Note that, in spite of cooling, the jet shock in the low-density collar (where $\eta<1$ ) must sink back into the body of the jet. The bow shock follows suit and the result is a highly oblique section of the shock in the inner collar. When undisturbed beam material impinges on this shock it is either directed toward the axis forming a strong pinch in the core or it is shunted radially outward forcing the outer collar to peel away. Thus in both the adiabatic, isothermal and radiative cases the nonuniformity in the jet shock drives a flow pattern that enhances the "jet within a jet" nature of the flow, and the core always ends up propagating away from the rest of the beam.

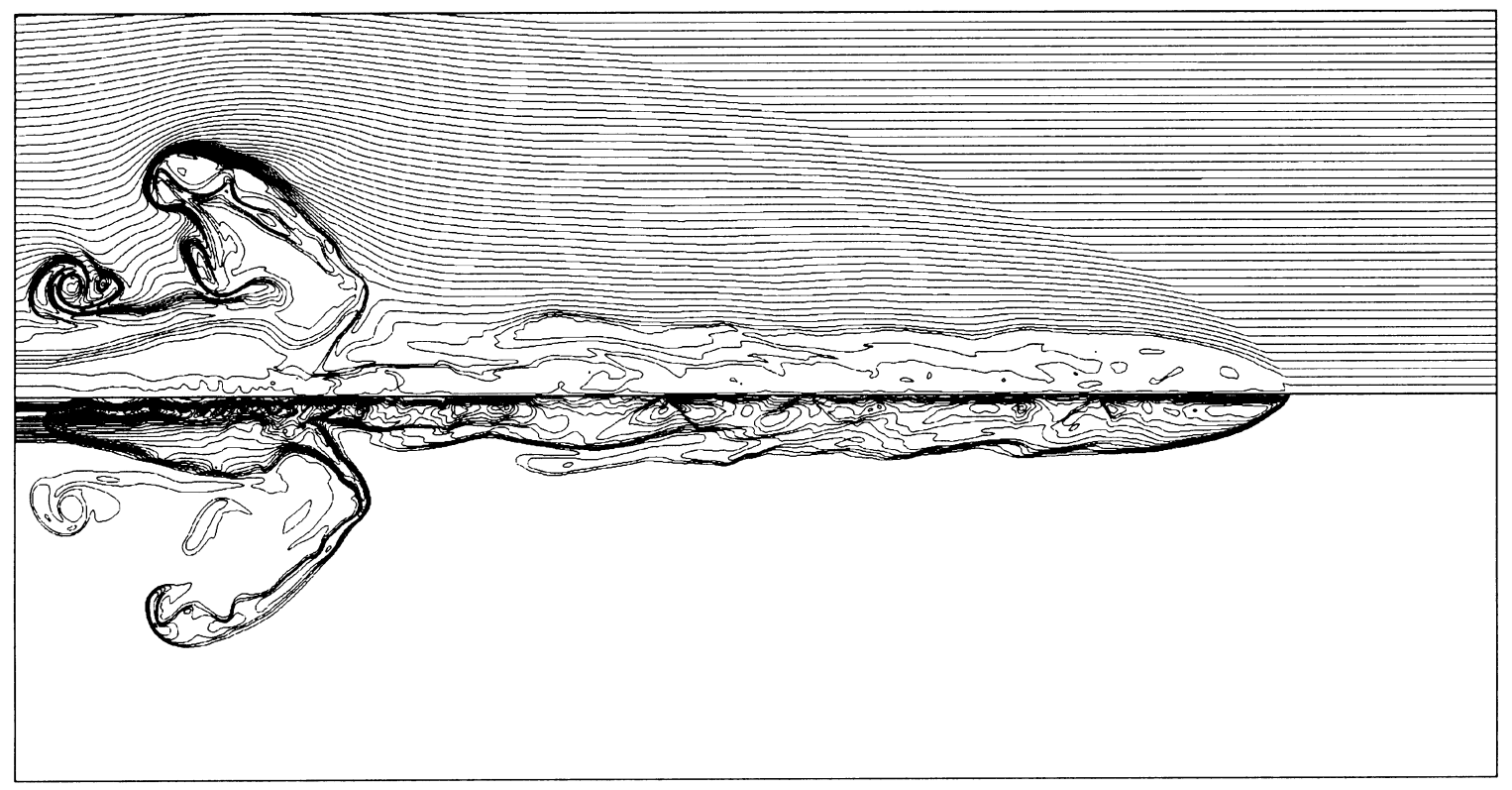

FIG. 13. - Magnetic field for multicomponent jet. Adiabatic simulation. Top half contour plot represents the poloidal field $\left(\boldsymbol{B}_{p}=B_{r} \hat{e}_{r}+B_{z} \hat{e}_{z}\right)$. The bottom half contour plot represents the toroidal field $\left(B_{\phi}\right)$. Note the increase in the magnetic pitch $B_{\phi} /\left|B_{p}\right|$ behind shocks. 


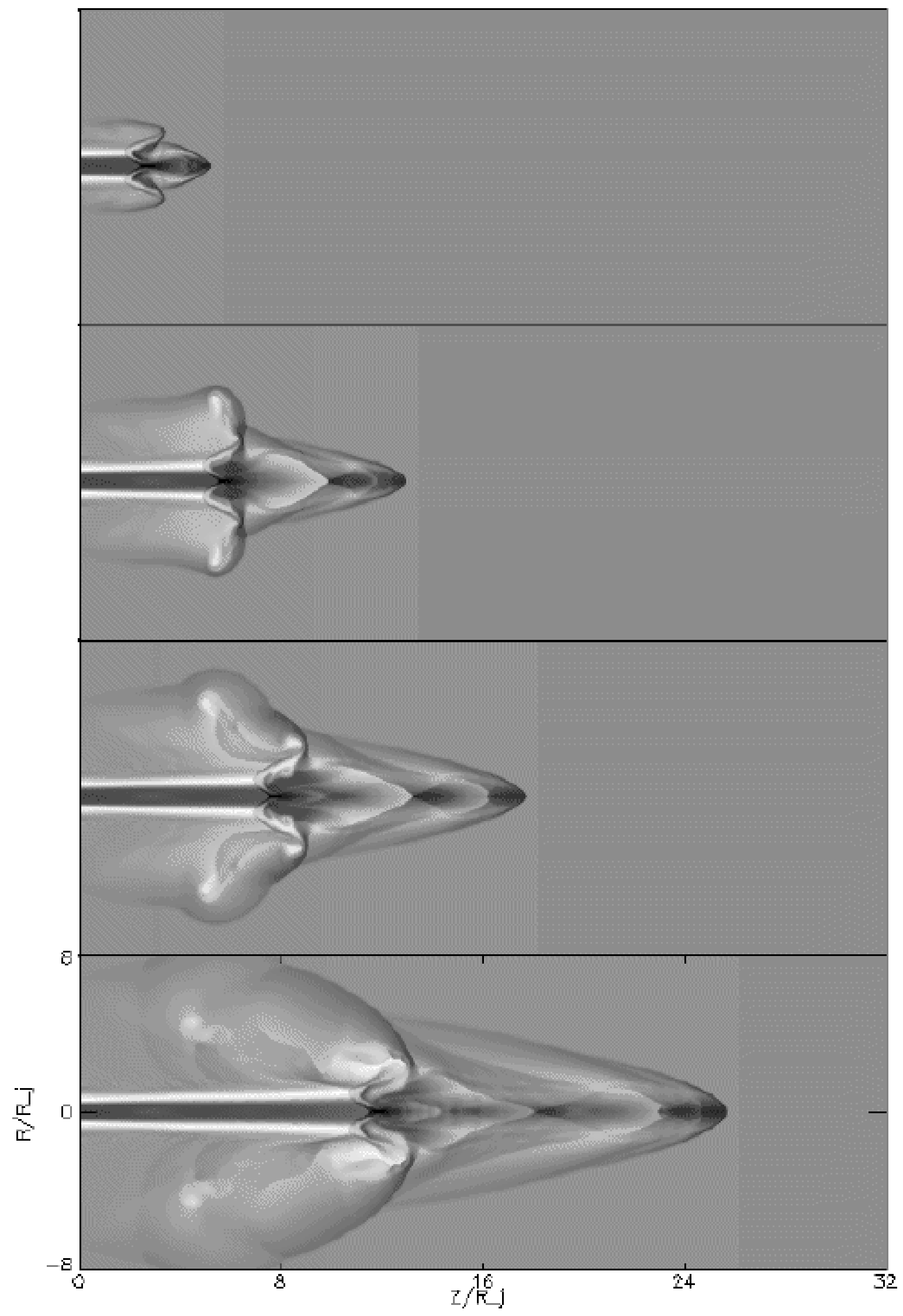

FIG. 14. - Gray-scale maps of density for radiative simulations of multicomponent jet. Four frames from the simulation are shown. 


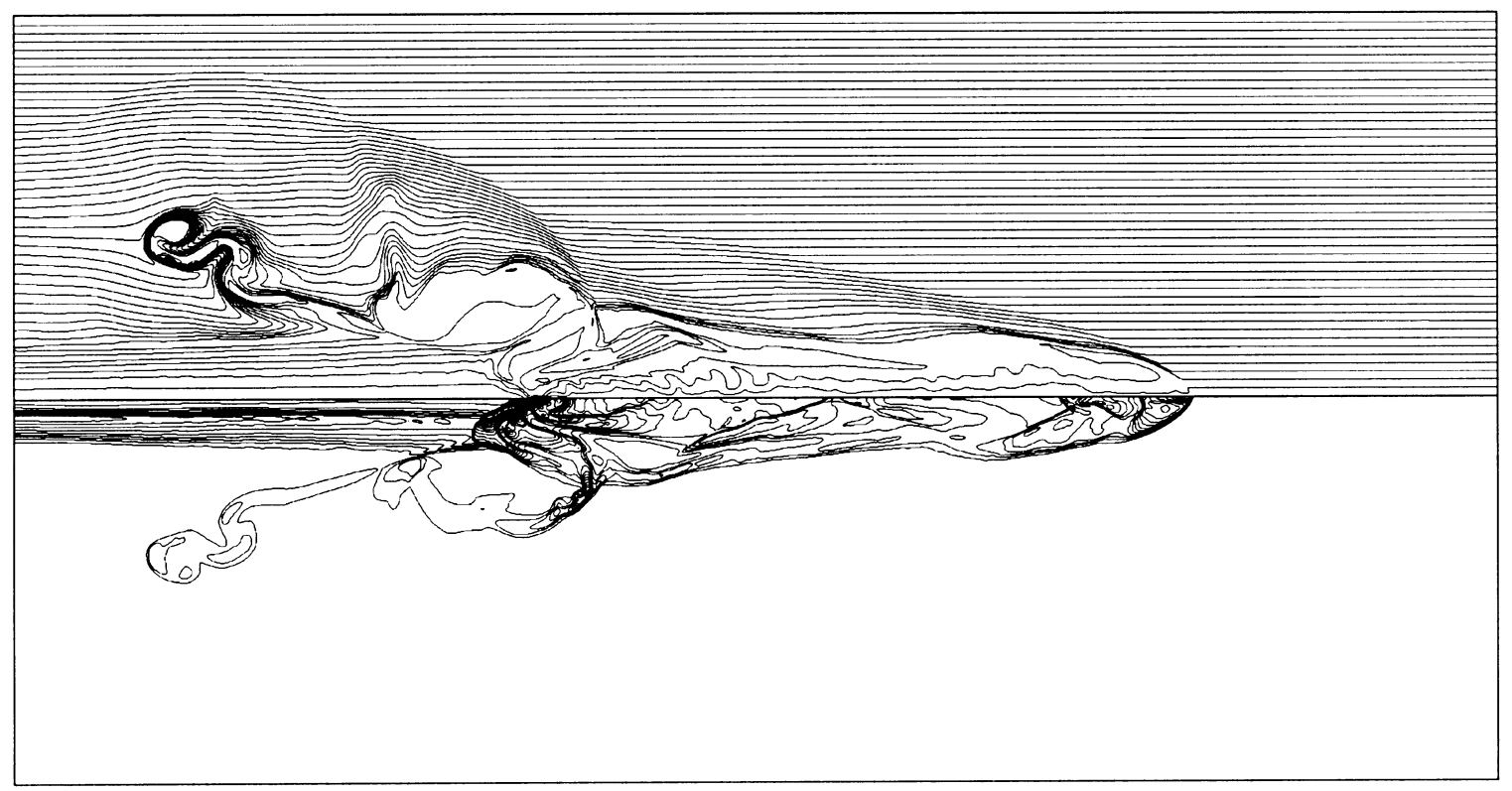

FIG. 15.-Magnetic field for multicomponent jet. Radiative simulation. The contour plot in the top half represents the poloidal field $\left(\boldsymbol{B}_{p}=B_{r} \hat{e}_{r}+B_{z} \hat{e}_{z}\right)$. The contour plot in the bottom half represents the toroidal field $\left(B_{\phi}\right)$. Note the increase in the magnetic pitch $B_{\phi} /\left|B_{p}\right|$ behind shocks.

Unlike the adiabatic model where the peel-off region had a low- $z$ velocity, both the radiative and isothermal models show the point at which the core and collar separate moves with a speed that is a large fraction of the beam speed. The origin of this effect appears to be the lower pressures behind the jet shock, which causes less deceleration. The propagation of the separation point may also be due to the reduced width of the bow shock and a smaller cocoon (both expected in nonadiabatic models).

The magnetic field structure shown in Figure 15 is similar to what is seen in the adiabatic case. Note, however, the strong pinch, which occurs at the point where the peel-off occurs. The loss of gas pressure support will also decrease $\beta$ implying that the toroidal field can now exert a stronger influence.

The principle difference between the radiative and isothermal models occurs in the core. First note that it is difficult to see the instabilities in the radiative model. A detailed inspection of the simulation frames shows they are present but they appear to diffuse more rapidly than in the isothermal case. The isothermal simulations do show the same form of the modes occurring as in the adiabatic models and with similar length scales. The difference between the isothermal and radiative solutions is likely due the greater thermalization that occurs in the higher Mach number flow.

\section{COMPARISONS WITH OBSERVATIONS}

In recent observations of molecular outflows (Dutrey, Guilloteau, \& Bachiller 1997; Gueth \& Guilloteau 1997, 1998) show small linear structures just ahead of the familiar bow-shaped shocks. Three examples of such features are presented in Figure 17. These structures point almost exactly away from the position of the protostellar condensation. These precursors of the bow shock show a roughly conical shape. As such, they could trace an underlying jet, which is propagating beyond the bow shock. The present

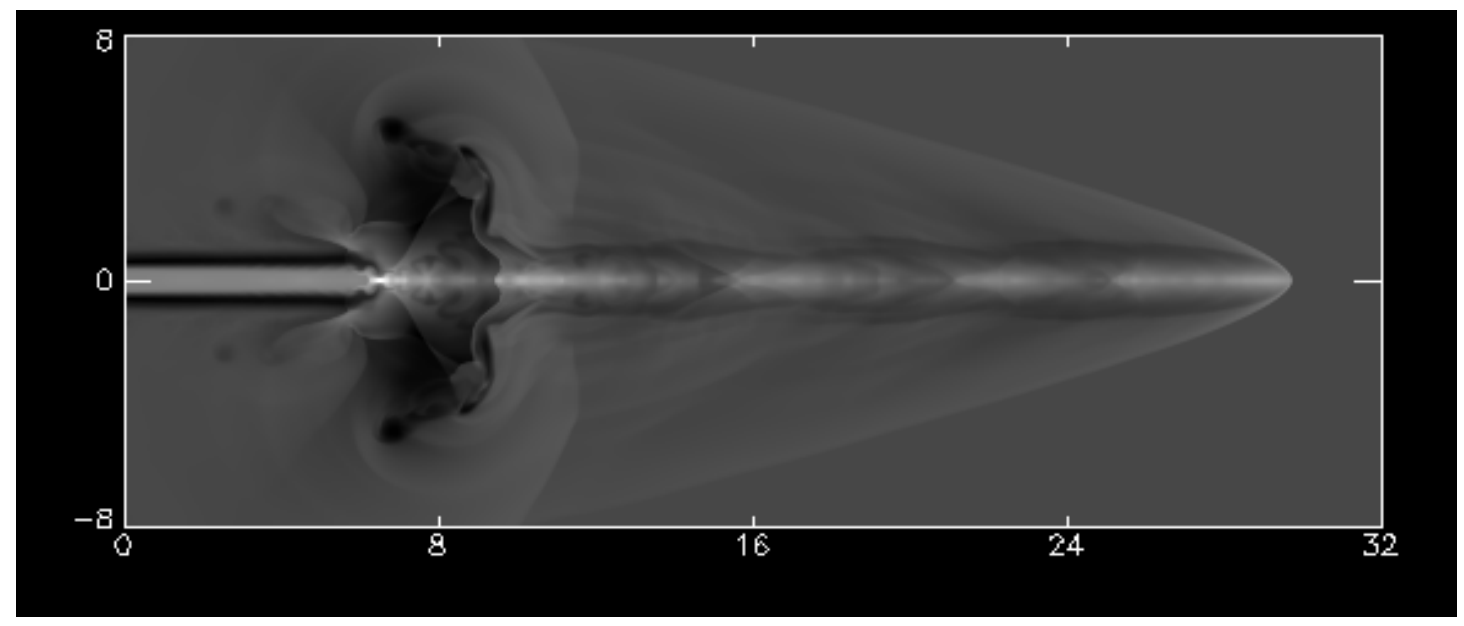

FIG. 16. - Gray-scale map of density for isothermal simulation of multicomponent jet. The simulation is shown at time $t=11.4$. 


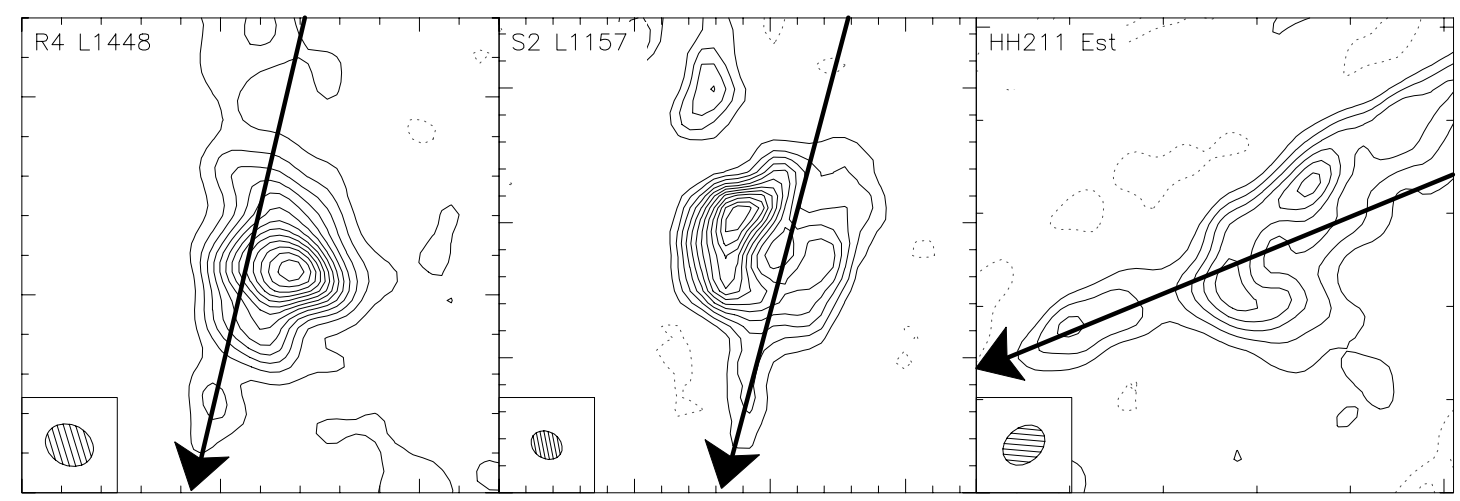

FIG. 17.- Observations of linear precursors: $\mathrm{SiO}(2-1)$ emission of L1448 (from Dutrey et al. 1997), $\mathrm{SiO}(2-1)$ emission of L1557 (from Gueth \& Guilloteau 1998), CO(2-1) emission of HH211 (from Gueth \& Guilloteau 1997) (courtesy of F. Gueth). The shocks exhibit an extension downstream, pointing away from the protostellar positions (thick lines). The arrow head marks direction of propagation.

simulations are suggestive offering an explanation for the observed structures. The fast core-jet propagates ahead of the collar and the surrounding molecular outflow.

The outer collar may be solely responsible for the larger bow shock structure or it may itself be embedded in a larger wide-angle wind. Thus molecular observations of conical precursors to the bow shocks may be a signature of density and magnetic stratification discussed in this study. Therefore, the global evolution that we obtain for our jets, e.g., a core-collar structure could lead to common behavior for several YSO jets, and also may help in understanding the relation between jets and molecular outflows.

\section{DISCUSSION AND CONCLUSIONS}

We have carried out a series of simulations intended to address the issue of MHD jet propagation. Whereas previous studies have used ad hoc initial conditions we inject flows into our computational grid derived from models of collimated jets driven by magnetocentrifugal launching. This strategy allows us to compare the propagation characteristics of jets driven by different types of outflows. In particular we have studied the propagation of jets driven by: (1) a purely Keplerian rotator (a disk) exterior to a solid body rotator (a star); (2) a Keplerian rotator with a subKeplerian boundary layer both of which are exterior to a solid body rotator. The former model we refer to as a Keplerian jet, the latter is called a multicomponent model. Our simulations follow the jets out to observable scales. In the Keplerian jet simulations the jet radius is $R_{j}=1.5$ $\times 10^{15} \mathrm{~cm}$ making the grid extend out to $3000 \mathrm{AU}$. For the multicomponent jet $R_{j}$ is almost a factor of 10 larger and the grid extends out $0.1 \mathrm{pc}$. The width of the multicomponent jet is interesting in that it yields a model with a very narrow, dense core (a jet) surrounded by a wider lower density outflow.

Both models were calculated under the assumption that the jets are launched under isothermal conditions. We have carried out simulations of the propagation of both Keplerian and Multicomponent jets under adiabatic, isothermal, and radiative conditions in order to determine the behavior of the resulting flows with, and without, radiative losses. We note again that our adiabatic and isothermal simulations have low magnetosonic Mach numbers $M_{\mathrm{ms}}=$ $2-4$. While these values are small compared with the values used in previous numerical studies of MHD jets $\left(M_{\mathrm{ms}}>10\right.$; Stone \& Hardee 2000) they is quite similar to what has been obtained in other studies MHD collimation of jets ( $M_{\mathrm{ms}} \approx 3$; Camenzind 1997).

Our simulations show significant differences in the propagation characteristics for the two types of rotators. In addition, features are seen in both classes of jet that have not been seen in previous models of either pure hydrodynamic or MHD jet propagation. In all cases it appears that the most important aspect of the flow behavior seen in the simulations can be traced back to the annular stratification of the jets. In particular, the radial distributions of density, velocity, and toroidal magnetic field appear to be the principle causes of the new behavior seen in the simulations. Both Keplerian and multicomponent jets exhibit a core/ collar structure such that a high-density core region exists near the axis surrounded by one or more lower density annuli (collar) extending out to the jet boundary. The strongest toroidal fields exist at the boundary between the core and collar.

Since the momentum in the core is higher than that in the collar the propagation characteristics of the jets are dominated by the core pulling ahead of the collar. The strong field surrounding the core ensures that the two regions remain fairly distinct in terms of their dynamics. As the jets propagate we see the core acting as a jet within a jet. In the Keplerian case the relatively low density contrast between core and collar keeps the two propagating at relatively similar velocities. The stratification of the magnetic fields produces strong dynamical differences between core and collar. All plasma flowing into the cocoon comes from the lower field strength regions of the collar. In the multicomponent case there exists an extremely low-density inner collar (which also has higher velocity than the surrounding regions) and this leads to a complete separation of core and collar. The "peel-off" of the collar in the multicomponent models is quite dramatic and occurs in both the adiabatic and isothermal simulations.

Our results have bearing on a number of issues. The simplest conclusion that can be drawn is that the structure imposed on a YSO jet by the launching and collimation process can lead to fairly complex propagation characteristics. Thus our models build on and extend the previous works, which utilized only "top-hat" jets as initial conditions. Our results also indicate that jets launched from different classes of rotators may have different propagation characteristics. It is likely that in real jet systems the dynamics is too complex to make an isomorphic identifica- 
tion of a given class of rotators with a set of observed jet morphologies. There is however the possibility that as these kinds of studies mature one might be able to distinguish between different classes of MHD launching models via consideration of the way the jets from these models would appear on the sky.

Finally we note that given the large parameter space of initial conditions for both the Given Geometry Model and for the jet propagation simulations, the work described here, which focuses only on two instances, must be seen as preliminary. It does however point to the fact that the jets produced by magnetized rotators are likely to be more complex in their structure and, furthermore, that this complexity will be reflected in the observed jet morphologies. In future studies we will attempt to build a larger catalog of jet propagation characteristics through a more thorough exploration of parameter space of the Given Geometry Model.

We wish to thank Guy Delemarter, Jack Thomas, Colin Norman, and Lee Hartmann for their input and discussions leading to this paper. This work was supported by NSF grant AST-0978765. D. R. was supported in part by KOSEF through grant 981-0203-0011-2.
Appl, S., Lery, T. \& Baty, H, 2000, A\&A, 355, 818

Bertout, C., Basri, G., \& Bouvier, J. 1988, ApJ, 330, 350

Burke, T., Mundt, R., \& Ray, T. P. 1988, A\&A, 200, 99

Camenzind, M. 1997, in IAU Symp. 182, Herbig-Haro Flows and the Birth of Low-Mass Stars, ed. B. Reipurth \& C Bertout (Dordrecht: Kluwer ), 241

Cerqueira, A. H., \& de Gouveia Dal Pino, E. M. 1999, ApJ, 510, 828

Cerqueira, A. H., de Gouveia Dal Pino, E. M., \& Herant, M. 1998, ApJ, 489, L185

Dalgarno, A., \& McCray, R. A. 1972, ARA\&A, 10, 375

Dutrey, A., Guilloteau, S., \& Bachiller, R. 1997, A\&A, 325, 758

Fendt, C., \& Camenzind, M. 1996, A\&A, 313, 591

Ferreira, J. 1997, A\&A, 319, 340

Frank, A., Gardiner, T., Delamarter, G., \& Betti R. 1999, ApJ, 524, 947

Frank, A., Ryu, D., Jones, T. W., \& Noriega-Crespo A. 1998, ApJ, 494, L79

Gardiner, T., \& Frank, A. 2000, ApJ, in press

Gardiner, T., Frank, A., Ryu, D., \& Jones, T. 2000, ApJ, 530, 834

Goodson, A, Winglee, R., \& Boehm, K. 1997, ApJ, 489, 199

Gueth, F., \& Guilloteau, S. 1997, A\&A, 325, 758 1998, A\&A, 333, 287

Harten, A. 1983, J. Comp. Phys., 49, 357

Hartmann, L. 1998, in Accretion Processes in Star Formation (Cambridge: Cambridge Univ. Press)

Heyvaerts, J., \& Norman, C. A. 1989, ApJ, 347, 1055

Hollenbach, D., \& McKee, R. 1979, ApJ, 41, 555

Kudoh, T., Matsumoto, R., \& Shibata, K. 1998, ApJ, 508, 186

Leahy, J. P. 1991, in Beams and Jets in Astrophysics, ed. P. Hughes (Cambridge: Cambridge Univ. Press), 100

Lery, T. 1996, Ph.D. thesis, ULP Strasbourg, France

Lery, T., \& Frank, A. 2000, ApJ, 533, 897

Lery, T., Henriksen, R. N., \& Fiege J. D. 1999a, A\&A, 350, 254

Lery, T., Heyvaerts, J., Appl, S., \& Norman, C. A. 1998, A\&A, 337, 603

\section{REFERENCES}

Lery, T., Heyvaerts, J., Appl, S., \& Norman, C. A. 1999b, A\&A, 347, 1055

Leveque, R. J. 1998, in Computational Methods for Astrophysical Fluid Flow (Springer: Berlin), 1

Lind, K., Payne, D., Meier, D., \& Blandford, R. 1989, ApJ, 344, 89

Ostriker, E. C. 1997, ApJ, 486, 291

Ouyed, R., \& Pudritz, R. E. 1997a, ApJ, 482, 712

Priest, E. 1986, Solar Magnetohydrodynamics (Dordrecht: Reidel), 199

Pudritz, R. E. 1991, in The Physics of Star Formation and Early Stellar Evolution, ed. C. J. Lada \& N. D. Kylafis ( NATO ASI Ser. C, 342; Dordrect: Kluwer), 365

Ramm, H. 1990, Fluid Dynamics for the Study of Transonic Flow (New York: Oxford Univ. Press)

Ray, T. P., Mundt, R., Dyson, J. E., Falle, S. A. E. G., \& Raga A. C. 1996, ApJ, 468, L103

Reipurth, B. 1997, in IAU Symp. 182, Herbig-Haro Flows and the Birth of Low-Mass Stars, ed. B. Reipurth \& C. Bertout (Dordrecht: Kluwer )

Romanova, M. M., Ustyugova, G. V., Koldoba, A.V., Chechetkin, V. M., \& Lovelace, R. V.E. 1998, ApJ, 500, 703

Ryu, D., \& Jones, T. W. 1995, ApJ, 442, 228

Ryu, D., Jones, T. W., \& Frank A. 1995a, ApJ, 452, 785

Ryu, D., Miniati, F., Jones, T. W., \& Frank, A. 1998, ApJ, 509, 244

Ryu, D., Yun, H. S., \& Choe, S. 1995b, J. Korean Astron. Soc., 28, 243

Sauty, C., \& Tsinganos, K. 1994, A\&A, 287, 893

Shu, F. H., Najita, J., Ostriker, E., \& Shang H. 1995, ApJ, 455, L155

Shu, F., Najita, J., Ostriker, E., Wilkin, F., Ruden, S., \& Lizano, S. 1994, ApJ, 429, 781

Soker, N., \& Livio, M. 1994, AJ, 421, 219

Stone, J., \& Hardee, P. 2000, ApJ, 540, 192

Strang, G. 1968, SIAMM J. Numer. Anal., 5, 506

Todo, Y., Uchida, Y., Sato, T., \& Rosner, R. 1992, PASJ, 44, 245

Trussoni, E., Tsinganos, K., \& Sauty, C. 1997, A\&A, 325, 1099

Vlahakis, N., \& Tsinganos, K. 1998, MNRAS, 298, 777 